\title{
ARITHMETICAL COMPOSITION AND INVERSION OF FUNCTIONS OVER CLASSES*
}

\author{
BY
}

E. T. BELL

\section{OvoIDS AND OVA}

1. If one or more of the postulates of a field be modified ( $\$ 13)$, contradicted ( $\$ 12)$, or suppressed ( $\$ 5)$, and if the altered set of postulates be selfconsistent, the set defines what we shall call a variety, which is abstract or special according as the marks in the postulates are arbitrary to the extent permitted by the postulates, or have specific interpretations (\$64). A special variety is an instance of the abstract variety from which it is obtained by specific interpretation, and the existence of an abstract variety is said to be demonstrated by exhibiting an instance.

2. The varieties in the present theory are among the most rudimentary possible; I have called them ovoids and ova. For certain of the classic postulate systems of a field the complete sets of conceivable varieties (obtained from the truth tables of the systems) exist; for others, not all varieties exist. For the moment it is sufficient to observe that a ring, not necessarily commutative, is an instance of the ovoids and ova defined presently. Hence all exist. On the other hand, none of these varieties is an instance of a ring, ray, or module, commutative or not, and hence all would appear to be new. They were not constructed ad hoc, but arose by necessity in the general algebra of numerical functions, of which they are the simple, abstract structure. It is noteworthy that a comprehensive theory, among whose instances are several extensive theories of numerical functions, many of which have themselves a high degree of generality, should be comprised in such narrow compass.

The principal definitions and postulates are in $\$ \S 3-6,9,15-21,33,42,44$, $45,58,60$, and the main conclusions in $\S \S 31,34-41,47-50,56-60,61-64$. The purpose of the theory is indicated in $\$ \$ 7,23,34,42$.

3. The notation $K \mid x, y, \cdots$ shall signify that the class $K$, different from the null class, contains the elements $x, y, \cdots$. To avoid separation into cases it is not assumed, unless so stated ( as in $\S \$ 15,16$ ), that the elements of a class are all distinct. Hence, unless the elements are stated to be all distinct and finite in number, $K$ will contain any finite number of elements.

\footnotetext{
* Presented to the Society, September 11, 1931; received by the editors May 4, 1931.
} 
A class will be called countable if it contains either only a finite number or a denumerable infinity of distinct elements.

4. Abstract equality, indicated by $=$, has its usual significance as a reflexive, symmetric, transitive relation, with the postulate (or theorem, as the case may be in a given context) that, if $K \mid x, y$, where $K$ is any non-null class, and if $x=y$, then either of $x, y$ may replace the other in any relation concerning $x$ or $y$ or both. The relation $\sim$ of $\$ 21$ is an instance of $=$, and is such, not by postulation, but by proof. All classes in the sequel are postulated to be such that equality is significant for the elements of any given class. The sign . . of implication will be used where convenient.

5. Capital Greek letters shall denote operations. $K$ is any non-null class, $t$ is an arbitrary constant integer $>0$.

A capital Latin superscript $O_{t}, C_{t}, P_{t}, O, C, P, B, A, D, \cdots$, as in $\Phi^{B} K$, connotes a property of $\Phi$ with respect to some (or all) one-row matrices whose elements are in $K$, and a formula (or compound symbol) of the type $\Phi^{I_{1} \cdots I_{r}} K$, where $I_{1}, \cdots, I_{r}$ denote properties, is an assertion. The like applies later to matrices of any numbers of rows and columns. The assertion $\Phi^{I_{1}} \cdots I_{r} K$ is the simultaneous assertion of $\Phi^{I_{i}} K(j=1, \cdots, r)$. If $\Phi^{I_{1} \cdots I_{r}} K$, we call $K$ a $\Phi$-ovoid of character $I_{1} \cdots I_{r}$. Formal definitions follow.

$\Phi^{O} K$ : asserts that the result $\left(x_{1}, \cdots, x_{t}\right)^{\Phi}$ of operating with $\Phi$ on the one-row matrix $\left(x_{1}, \cdots, x_{t}\right)$ is uniquely known whenever $K \mid x_{1}, \cdots, x_{t}$ and $\left(x_{1}, \cdots, x_{t}\right)$ is given. If $t=1$, by convention* $(x)^{\Phi}=x^{\Phi}=x$ whenever $K \mid x$.

$\Phi^{C_{t}} K$ : asserts $\Phi^{O_{t}} K$ and $K \mid(x, \cdots, x)^{\Phi}$ whenever $K \mid x_{1}, \cdots, x_{t}$ and the matrix $\left(x_{1}, \cdots, x_{t}\right)$ is given.

$\Phi^{P_{t}} K$ : asserts that $\left(x_{1}, \cdots, x_{t}\right)^{\Phi}$ is a symmetric function of $x_{1}, \cdots, x_{t}$ whenever $\Phi^{O_{t}} K$ and $\left(x_{1}, \cdots, x_{t}\right)^{\Phi}$ is defined.

$\Phi^{O} K$ : asserts $\Phi^{O}{ }^{O} K$ for all finite integers $t>0$;

$\Phi^{C} K$ : asserts $\Phi^{C}{ }^{t} K$ for all finite integers $t>0$;

$\Phi^{P} K$ : asserts $\Phi^{P} t K$ for all finite integers $t>0$;

$\Phi^{B} K$ : asserts $\Phi^{C} K$ and that

$$
\left(x_{1}, \cdots, x_{r}, x_{r+1}\right)^{\Phi}=\left(\left(x_{1}, \cdots, x_{r}\right)^{\Phi}, x_{r+1}\right)^{\Phi}
$$

for all integers $r>0$, whenever $x_{1}, \cdots, x_{r}, x_{r+1}$ are in $K$ and $\left(x_{1}, \cdots, x_{r}\right.$, $\left.x_{r+1}\right)^{\Phi},\left(x_{1}, \cdots, x_{r}\right)^{\Phi}$ are defined. By convention, if $\Phi^{B} K$, then $(x)^{\Phi}=x^{\Phi}=x$ whenever $K \mid x$. This convention as a separate statement is unnecessary, by the previous convention, but is included on account of its importance.

* The restriction on $\Phi$ imposed by this convention is only apparent, and is a mere convenience of notation. The important case where the convention need not hold is provided for in the discussion of functions in $\$ 21$, for which a different notation is used. 
$\Phi^{A} K:$ asserts $\Phi^{B} K$ and that

$$
\left((x, y)^{\Phi}, z\right)^{\Phi}=\left(x,(y, z)^{\Phi}\right)^{\Phi}
$$

whenever $K \mid x, y, z$ and $(x, y, z)$ is given.

$(\Psi, \Phi)^{D} K$ : asserts $\Psi^{B} K$ and $\Phi^{B} K$, and that

$$
\left((x, y)^{\Psi}, z\right)^{\Phi}=\left((x, z)^{\Phi},(y, z)^{\Phi}\right)^{\Psi}
$$

whenever $K \mid x, y, z$ and $(x, y, z)$ is given.

The compound assertion $\Phi^{A} K$ and $K \mid x, \cdots$ will be written $\Phi^{A} K \mid x, \cdots$, and likewise in all similar situations.

6. Several modifications of the preceding definitions lead to effectively the same conclusions. For example, in defining $\Phi^{B} K$, it is sufficient to postulate $\Phi^{O} K$, or even $\Phi^{O_{2}} K$, instead of the much stronger $\Phi^{C} K$, as the clause following and implies $\Phi^{c} K$ when $\Phi^{o} K$. The foregoing however are in their most convenient form for our purpose.

If desirable to describe the processes and properties just defined, the following will be adopted: $O$, over or open, signifying that closure $C$ of $K$ under $\Phi$ is not postulated; $C$, closed; $P$, totally permutable, or totally commutative; $P_{2}$, commutative; $B$, binary; $A$, associative; $D,(\Psi, \Phi)$-distributive; a suffix $t$ on a property adds the restriction of order $t$; if $\Phi^{c} K$ is postulated we say that $\Phi$ is on $K$; if $\Phi^{o} K, \Phi$ is over $K$.

If $\left(x_{1}, \cdots, x_{r}\right)^{\Phi}$ is defined, we call it the $\Phi$-composite of $\left(x_{1}, \cdots, x_{r}\right)$, and say that $\left(x_{1}, \cdots, x_{r}\right)^{\Phi}$ has been derived from $\left(x_{1}, \cdots, x_{r}\right)$ by $\Phi$-composition; the $\Phi$-components of $\left(x_{1}, \cdots, x_{r}\right)^{\Phi}$, in this order, are $x_{1}, \cdots, x_{r}$.

When two operations are connected by a property for whose definition both are necessary, as in the definition of $D$, the class $K$ is called an ovum with respect to the two operations in a prescribed order.*

7. One of the problems with which we shall be concerned can now be stated. Given ovoids and ova of prescribed characters, to construct from them further operations and classes of values of functions such that with respect to the new operations the new classes shall be varieties abstractly identical with those occurring in the algebra of numerical functions. The sense in which inversion is used in this theory is explained in $\$ 23$.

The solution obtained here is extremely general. It includes all known algebras of numerical functions, unifies them by demonstrating their abstract identity, exhibits them as simple instances of the abstract theory constructed, and provides the means for obtaining an indefinite number of further in-

* I introduced the term ovum in a previous paper, American Mathematical Monthly, vol. 37 (1930), p. 400. A note in the same volume, p. 484, may be glanced at in connection with the present paper. See also $\$ 62$. 
stances. A point of particular interest is the formal identity of the abstract theory, for functions of any finite number of general variables, with the classical algebra of either power series or Dirichlet series of a single numerically valued variable or with the like for $r$ variables. The principal question propounded in the second paper cited in the footnote is therefore answered in the negative, in spite of the very different appearances which particular solutions present. Further generalizations will be indicated as we proceed, by suggesting weakened hypotheses which lead to similar but more general conclusions.

Whenever, as in parts of $\$ 8$, an assertion is an immediate consequence of the definitions or of what has preceded, proof will be omitted without comment. For example, $\Phi^{C} K$ does not imply $\Phi^{B} K$, but $\Phi^{B} K$. د. . $\Phi^{C} K$.

8. If $\Phi^{I_{1} \cdots I_{r}} K$ is significant, so also are $\Phi^{I_{i}} K(i=1, \cdots, r)$, and $\Phi^{I_{1} \cdots I_{r}} K$. כ. $\Phi^{I_{i}} K(i=1, \cdots, r)$. By this remark the following columns may be extended. We collect for easy reference the simplest consequences of the definitions in $\$ 5$ that will be most frequently used, generally without further reference. By $\Phi^{I_{1}+\cdots+I_{r}} K$ we assert $\Phi^{I_{1}} K$ or $\Phi^{I_{2}} K, \cdots$, or $\Phi^{I_{r}} K$, and the statement that $\Phi^{I} K . \supset . \Phi^{I_{1}+\cdots+I_{r}} K$ is false is the assertion that all of $\Phi^{I} K . \supset . \Phi^{I_{i}} K(i=1, \cdots, r)$ are false.

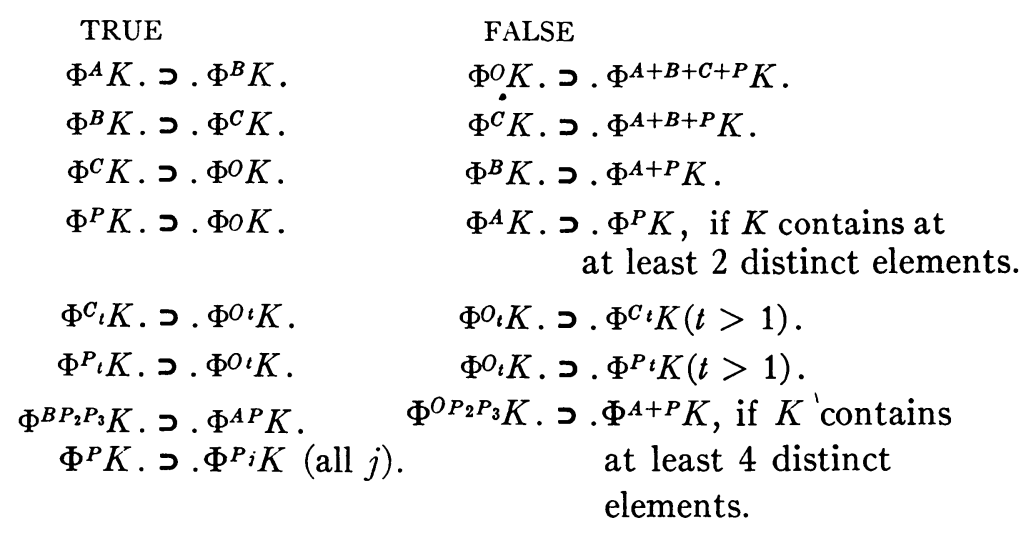

We prove $\Phi^{B P_{2} P_{3}} K$. כ. $\Phi^{A P} K$. It will suffice to prove the $A$ part, as the rest follows, precisely as may be proved in a field, from $\Phi^{B A P_{2}} K . \supset . \Phi^{P} K$.

Let $\Phi K \mid(x, y, z)^{\Phi}$. Then

Hence

$$
\begin{aligned}
& \Phi^{B} K \text {. ว . }(x, y, z)^{\Phi}=\left((x, y)^{\Phi}, z\right)^{\Phi} ; \\
& \Phi^{B P_{3}} K \text {. ว . }(x, y, z)^{\Phi}=(y, z, x)^{\Phi}=\left((y, z)^{\Phi}, x\right)^{\Phi} \text {; } \\
& \Phi^{B P_{2}} K \text {. כ . }\left((y, z)^{\Phi}, x\right)^{\Phi}=\left(x,(y, z)^{\Phi}\right)^{\Phi} \text {. }
\end{aligned}
$$

$$
\Phi^{B P_{2} P_{3}} K \mid(x, y, z)^{\Phi} . \text { ว . }\left((x, y)^{\Phi}, z\right)^{\Phi}=\left(x,(y, z)^{\Phi}\right)^{\Phi},
$$

which completes the proof. 
A useful property implied by $\Phi^{A} K$ is the following. If

then

$$
\Phi^{A} K \mid x_{1}, \cdots, x_{r}, y_{1}, \cdots, y_{s}, \cdots, z_{1}, \cdots, z_{t}
$$

$$
\begin{aligned}
& \left(\left(x_{1}, \cdots, x_{r}\right)^{\Phi},\left(y_{1}, \cdots, y_{s}\right)^{\Phi}, \cdots,\left(z_{1}, \cdots, z_{t}\right)^{\Phi}\right)^{\Phi} \\
& \quad=\left(x_{1}, \cdots, x_{r}, y_{1}, \cdots, y_{s}, \cdots, z_{1}, \cdots, z_{t}\right)^{\Phi} .
\end{aligned}
$$

If $\Phi^{A} K \mid x$, we shall write $(x, \cdots, x)^{\Phi}=x^{r \Phi}$ (precisely $r x^{\prime}$ s in first). Hence

$$
\Phi^{A} K \mid x . \supset .\left(x^{r \Phi}, x^{s \Phi}\right) \Phi=x^{(r+s) \Phi} .
$$

9. Of the many possible kinds of $(\Psi, \Phi)$-ova, defined by $D$ and specific properties of $\Psi, \Phi$, from which we may construct compositions of functions, we shall consider only one. If

$$
(\Psi, \Phi)^{D} K, \text { and } \Psi^{C A P} K, \text { and } \Phi^{C A P} K,
$$

we shall call $K$ a double $(\Psi, \Phi)$-ovum, and write $(\Psi, \Phi)^{C A P D} K$. By $\S 8$ this definition contains several redundancies. But as it is in the form most often applied, we shall not restate the hypotheses on $\Psi, \Phi$ in terms of their weakest equivalents.

If $K$ is a double $(\Psi, \Phi)$-ovum, and if $K \mid x, y, z$, then $K$ contains all of the following, which are equal:

$$
\left((x, y)^{\Psi}, z\right)^{\Phi},\left((x, z)^{\Phi},(y, z)^{\Phi}\right)^{\Psi},
$$

and the elements obtained from these by interchanging the symbols within ()$^{\Psi},()^{\Phi}$ respectively in all possible ways.

10. A commutative ring is an instance of a double $(\Psi, \Phi)$-ovum, but not conversely, as $\Psi$ is not assumed to have an inverse, and neither $\Psi$ nor $\Phi$ is postulated to have a modulus. For the same reason a double $(\Psi, \Phi)$-ovum is not a commutative group with respect to either $\Psi$ or $\Phi$, nor is it a $\Psi$ or $\Phi$ commutative semigroup, since there is no postulate of cancellation.

The following device is useful in supplying details of proofs in complicated situations concerning double ova. By what has just been remarked, if $K$ is a double $(\Psi, \Phi)$-ovum, $(\Psi, \Phi)$ may be replaced by the $(+, X)$ of a commutative ring, provided that neither the existence of an inverse to $\Psi$ nor that of moduluses with respect to either $\Psi$ or $\Phi$ be assumed.

A similar device, with the obvious greater restrictions necessary, applies. to ovoids of prescribed characters, say $A, P, C P, A P$, compared with modules, rings, rays, groups and semigroups. By the indicated changes of notation, classical proofs can be transferred directly to ovoids and ova, and there is no need to reproduce them; after having verified that the above proviso, or the necessary equivalent for a particular character, is not violated. 
11. Applying $\$ 10$, we readily see the following, which will be required later. Let $K$ be a double $(\Psi, \Phi)$-ovum, and let $K$ contain all of

$$
\begin{gathered}
a_{\lambda_{1}}, \cdots, a_{\lambda r} ; b_{\mu 1}, \cdots, b_{\mu s} ; \cdots ; c_{\nu 1}, \cdots, c_{\nu t} \\
(\lambda=1, \cdots, l) ;(\mu=1, \cdots, m) ; \cdots ;(\nu=1, \cdots, n) .
\end{gathered}
$$

Then the $\Phi$-composite

$$
\begin{array}{r}
{\left[\left\{\left(a_{11}, \cdots, a_{1 r}\right)^{\Phi}, \cdots,\left(a_{l 1}, \cdots, a_{l r}\right)^{\Phi}\right\}\right.} \\
\left\{\left(b_{11}, \cdots, b_{1 s}\right)^{\Phi}, \cdots,\left(b_{m 1}, \cdots, b_{m s}\right)^{\Phi}\right\} \\
\cdots \\
\cdots \\
\left\{\left(c_{11}, \cdots, c_{1 t}\right)^{\Phi}, \cdots,\left(c_{n 1}, \cdots, c_{n t}\right)^{\Phi}\right\} \Psi
\end{array}
$$

is equal to the $\Psi$-composite of the $l m \cdots n \Phi$-composites

$$
\begin{gathered}
\left(a_{\lambda 1}, \cdots, a_{\lambda r}, b_{\mu 1}, \cdots, b_{\mu s}, \cdots, c_{\nu 1}, \cdots, c_{\nu t}\right)^{\Phi} \\
(\lambda=1, \cdots, l ; \mu=1, \cdots, m ; \cdots ; \nu=1, \cdots, n) .
\end{gathered}
$$

12. The distinction between suppressing a postulate and contradicting it leads to the consideration of disjunctive varieties. Let $R^{\prime}$ denote the negation of the property $R$; for example, $A^{\prime} \equiv$ non-associative. Let $R, Q, \cdots$ be any properties significant for $\Phi$ when $\Phi^{o} K$. Then if at least one but not all of $R^{\prime}, Q, \cdots$ is a negation, and $\Phi^{R^{\prime} Q \cdots} K$, we call $K$ a $\Phi$-ovoid of disjunctive character $R^{\prime} Q \cdots$. The extension to more than one operation is obvious and need not be formally stated.

There are at least 32 disjunctive ovoids in the literature of postulate systems for groups and fields, and many more exist. These will be referred to in $\$ 64$. For some of the classical postulate systems for a field, the complete sets of disjunctive ovoids characterized by the truth tables of the systems exist; for others of these systems there is only partial existence.

From an ovoid with a given disjunctive character, a theory parallel to that developed in the sequel can be constructed. According to the specific characters, these theories will be either more or less general than that constructed here, which is based on ovoids and double ova; none of the possible theories has precisely the same degree of generality as this one. I have worked out several, but a complete discussion is beyond any reasonable limits of a single paper.

13. The sense in which a postulate is modified, as stated in $\S 1$, is as follows. If a postulate is a compound assertion, the compound is to be replaced by the simple predications of which it is composed. For example, "addition has a unique modulus," is to be replaced by "addition has a modulus" and "the modulus is unique." When so decomposed, the postulates are then to be 
contradicted or suppressed in all possible ways. There thus appear to be not more than $2^{12}-1$ conceivable varieties, of which all but a possible maximum of 1152 are non-existent.*

Some of the less well known extant varieties, for example an ovoid with character $A^{\prime} C$, give rise to striking algebras of numerical functions.

The theory based on ovoids and double ova outlined here is closer than any of the others to the algebra of numerical functions in rational arithmetic. As it includes all of the known algebras of numerical functions and produces an infinity more, it is sufficient for the present.

\section{II. $\Omega$-NUMBERS AND FUNCTIONS}

14 . As the $\Omega$-numbers now introduced are a new species of number, and as they are basic for the sequel, we shall describe their nature in detail. It is to be noticed that as first defined in $\S 15$, the only property of $\Omega$ assumed is that its component operations shall be respectively over $r$ arbitrary classes according to the general definition of $\Phi^{O} K$ in $\S 5$. If the operations be specialized by the imposition of further properties, so that the elements of the $\Omega$-number are restricted to be in varieties of prescribed characters, for example, if the elements are positive rational integers, we obtain the numbers necessary for composition over varieties having determinate characters. Thus the procedure to be followed in constructing any of the possible theories mentioned in $\$ \S 7,12,13$ will be evident from the present ones, which have the common character $A$. The numbers defined in $\$ 15$ are generalized in $\$ \S 50$, $52,53,58$.

15. An $\Omega$-number $Z$ of order $r$, degree $h$, and index $(r, h)$ is a matrix of $r$ rows and $h$ columns, whose $r h$ elements belong to any $r$ countable classes $K_{1}, \cdots, K_{r}$, of the following kind: the $h$ elements in the $j$ th row of $Z$ belong to $K_{j}$, and it is postulated that there exist $r$ operations $\Omega_{j}$ such that $\Omega_{j}{ }^{o} K_{j}(j=1, \cdots, r)$.

The $\Omega$ from which the numbers take their name is the one-column matrix

$$
\Omega \equiv\left\|\begin{array}{c}
\Omega_{1} \\
\vdots \\
\Omega_{r}
\end{array}\right\|,
$$

interpreted as an operation over the class $J$ of $\Omega$-numbers of order $r$. The result $Z^{\Omega}$ of operating with $\Omega$ on $Z$ is defined to be the one-column matrix of $r$ rows in which the element in the $j$ th row is the $\Omega_{j}$-composite (\$6) of the $h$

* For these calculations I am indebted to Mr. C. R. Worth, whose investigation, when complete, will exhibit the totality of varieties that exist. See also $\$ 64$. 
elements in the $j$ th row of $Z(j=1, \cdots, r)$. Thus, if $Z$ is defined by the first of the following matrices, the second defines $Z^{\Omega}$ :

$$
\left\|\begin{array}{c}
z_{11}, \cdots, z_{1 h} \\
\cdot . \cdot \cdot \cdot \\
z_{r 1}, \cdots, z_{r h}
\end{array}\right\|, \quad\left\|\begin{array}{c}
\left(z_{11}, \cdots, z_{1 h}\right)^{\Omega_{1}} \\
\cdot . \cdot . \cdot . \\
\left(z_{r 1}, \cdots, z_{r h}\right) \Omega_{r}
\end{array}\right\| .
$$

If, as above, $J$ denotes the class of all $\Omega$-numbers, and if $Q$ is a character such that $\Omega_{j}{ }^{Q} K(j=1, \cdots, r)$, then $\Omega^{Q} J$.

Since $\Omega$-numbers of index $(r, h)$ are matrices of $r$ rows and $h$ columns, equal and distinct $\Omega$-numbers of index $(r, h)$ are automatically defined, and similarly for the $\Omega$-decompositions introduced in a moment.

If each of $\Omega_{j}(j=1, \cdots, r)$ has the character $Q R \cdots S$, so that

$$
\Omega_{j}{ }^{Q R} \cdots s K_{j} \quad(j=1, \cdots, r),
$$

we say that $\Omega$ has the character $Q R \cdots S$, with respect to $\left(K_{1}, \cdots, K_{r}\right)$ if necessary. The only character of $\Omega$ so far is $O$.

With the notation as above for $Z, Z^{\Omega}$, let $\Omega$ now have the character $C(\$ 5)$. Then $Z^{\Omega}$ is an $\Omega$-number, say

$$
Z^{\Omega}=\left\|\begin{array}{c}
z_{1} \\
\vdots \\
z_{r}
\end{array}\right\|, \quad K_{j} \mid z_{j} \quad(j=1, \cdots, r) .
$$

If, conversely, $\Omega$ has the character $C$, and if an $\Omega$-number $X$ of index $(r, 1)$ be given, say

$$
X=\left\|\begin{array}{c}
x_{1} \\
\cdot \\
\dot{x}_{r}
\end{array}\right\|, \quad \Omega_{j}^{c} K_{j} \mid x_{j} \quad(j=1, \cdots, r)
$$

and if further there exists an $\Omega$-number $X_{(h)}$ of index $(r, h)$,

$$
X_{(h)} \equiv\left\|\begin{array}{c}
x_{11}, \cdots, x_{r h} \\
\cdot . \cdot \cdot \cdot \\
x_{r 1}, \cdots, x_{r h}
\end{array}\right\|, \Omega_{j}{ }^{c} K_{j} \mid x_{j i}(j=1, \cdots, r ; i=1, \cdots, h),
$$

such that $X_{(h)}^{\Omega}=X$, we call $X_{(h)}$ an $\Omega$-decomposition of index $(r, h)$ of $X$.

The class of all distinct $\Omega$-decompositions of index $(r, h)$ of $X$ is called the total $\Omega$-decomposition of index $(r, h)$ of $X$. Since the order $r$ will be fixed in a given context unless otherwise noted (\$59), we may refer only to the degree $h$, and speak of total $\Omega$-decompositions of degree $h$. These definitions have con- 
tent if and only if $\Omega$ has the character $C$. The possible generalization where $C$ is not postulated for $\Omega$ is not considered in this paper; it presents no difficulty. Since $A . \supset . C$, the definitions are significant if $\Omega$ has the character $A$.

16. We shall assume henceforth the following

Postulate. The total $\Omega$-decomposition of index $(r, h)$ of an $\Omega$-number of index $(r, 1)$ contains only a finite number of elements (distinct $\Omega$-decompositions of index $(r, h))$.

It is on account of this postulate that the present theory is said to be arithmetical. The reasons for our assumption will appear as we proceed. In composition of functions over classes, $\Omega$-decompositions of degree $h$ play a part abstractly identical with that of the resolution in all distinct ways (order relevant) of a positive integer into a product of $h$ positive integers. For this isomorphism to have a meaning, the postulate is necessary.*

17. Returning to $\$ 15$, we define $\left(K_{1}, \cdots, K_{r}\right) \equiv\left(K^{r}\right), r$ superscript, to be the class of all one-row matrices $\left(z_{1}, \cdots, z_{r}\right)$ generated as $z_{i}$ ranges over all elements of $K_{i}(i=1, \cdots, r)$. If $z_{i}^{\prime}$ is a particular element of $K_{i}$, and $z_{i}$ a variable element of $K_{i}(i=1, \cdots, r)$, we call $\left(z_{1}^{\prime}, \cdots, z_{r}^{\prime}\right)$ a value of the matric variable $\left(z_{1}, \cdots, z_{r}\right)$ of order $r$ over $\left(K^{r}\right)$. Thus $\left(K^{r}\right)$ is the class of all values of the matric variable $\left(z_{1}, \cdots, z_{r}\right)$.

When, as in $\$ 15, \Omega_{i}{ }^{o} K_{i}(i=1, \cdots, r)$, we shall write $\dagger\left(\Omega^{O} K^{r}\right)$. Similarly, if $\Omega_{i}{ }^{Q} K_{i}(i=1, \cdots, r)$, we write $\left(\Omega^{Q} K^{r}\right)$, and, if necessary to emphasize the character $Q$, we shall refer to $\Omega^{Q}$-numbers and $\Omega^{Q}$-decompositions.

If for the moment $L$ denotes the class of all matric variables of order $r$, and $Q$ is any character, then

$$
\left(\Omega^{Q} K^{r}\right) . \supset . \Omega^{Q} L \text {. }
$$

18. The notation being as in $\$ 15$, we write for the moment

$$
\left(x_{1 j}, \cdots, x_{r j}\right) \equiv X_{j}^{\prime} \quad(j=1, \cdots, h) .
$$

The one-row matrix $\left(X_{1}^{\prime}, \cdots, X_{h}^{\prime}\right)$, whose $h$ elements are the one-row matrices $X_{1}^{\prime}, \cdots, X_{h}^{\prime}$ in this order, namely the transposes of the columns of $X_{(h)}$ in their order from left to right, is called the transfer of the $\Omega$-number $X_{(h)}$ of index $(r, h)$. If $h=1$, transfer and transpose coincide; if $h>1$, there is no correspondent of the transfer in the classical algebra of matrices.

\footnotetext{
* If the postulate is not assumed, the corresponding theory applies to the field of all algebraic numbers, among others, but appears to be of only artificial interest.

$\dagger$ By $\S 5$, it would be meaningless to write $\Omega^{O}\left(K^{r}\right)$, as $\Omega$ has not been defined as an operation over elements of $\left(K^{r}\right)$, but as an operation on $\Omega$-numbers. An operation $\Omega^{\prime}$ is defined in $\S 41$ such that $\Omega^{\prime} O\left(K^{r}\right)$. When and only when $r=1, \Omega^{\prime} O\left(K^{r}\right)$ and $\left(\Omega^{O} K^{r}\right)$ have the same content.
} 
The transfer of the $X_{r}$ in $\$ 15$ is the matric variable $\left(x_{1}, \cdots, x_{r}\right)$.

The relation of transference is reciprocal in the sense of the following definition. We say that, conversely, $X_{(h)}$ as in $\$ 15$ is the transfer of $\left(X_{1}^{\prime}, \cdots, X_{h}^{\prime}\right)$. Hence $X$ as in $\$ 15$ is the transfer of $\left(x_{1}, \cdots, x_{r}\right)$.

19. The following definitions are fundamental in the theory of composition. In the notation of $\$ 18,\left(X_{1}^{\prime}, \cdots, X_{h}^{\prime}\right)$ is called an $\Omega$-decomposition of index $(r, h)$ of the matric variable $\left(x_{1}, \cdots, x_{r}\right)$.

The total $\Omega$-decomposition of index $(r, h)$ of the matric variable $\left(x_{1}, \cdots, x_{r}\right)$ is the class whose elements are the transfers of the elements in the total $\Omega$-decomposition of index $(r, h)$ of the transfer of $\left(x_{1}, \cdots, x_{r}\right)$.

20. The conjoint $* X, \cdots, Z \|$ of any number of $\Omega$-numbers $X, \cdots, Z$ of the same degree $r$,

$$
X \equiv\left\|\begin{array}{c}
x_{11}, \cdots, x_{1 a} \\
\cdot . \cdot . \cdot . \\
x_{r 1}, \cdots, x_{r a}
\end{array}\right\|, \cdots, Z \equiv\left\|\begin{array}{c}
z_{11}, \cdots, z_{1 c} \\
\cdot . \cdot \cdot \cdot] \\
z_{r 1}, \cdots, z_{r c}
\end{array}\right\|,
$$

in the order $X, \cdots, Z$, is the $\Omega$-number $W$, where

$$
W \equiv\left\|\begin{array}{c}
x_{11}, \cdots, x_{1 a}, \cdots, z_{11}, \cdots, z_{1 c} \\
\cdot . \cdot \cdot \cdot \cdot \cdot . \cdot . \cdot . \\
x_{r 1}, \cdots, x_{r a}, \cdots, z_{r 1}, \cdots, z_{r c}
\end{array}\right\| .
$$

If $X^{\prime}, \cdots, Z^{\prime}, W^{\prime}$ are the transfers of $X, \cdots, Z, W$ above, the conjoint $\left(X^{\prime}, \cdots, Z^{\prime}\right)$ of $X^{\prime}, \cdots, Z^{\prime}$, in this order, is $W^{\prime}$.

From the last of $\S 8$ it follows that if $\left(\Omega^{A} K^{r}\right)$, and $W \equiv\|X, \cdots, Z\|$, where $X, \cdots, Z$ are $\Omega^{A}$-numbers, then

$$
W^{\Omega} \equiv\|X, \cdots, Z\|^{\Omega}=\left\|X^{\Omega}, \cdots, Z^{\Omega}\right\| \Omega,
$$

and $W^{\Omega}$ is of degree 1 . If $\left(\Omega^{A} K^{r}\right)$ be weakened to $\left(\Omega^{B} K^{r}\right)$, or modified to $\left(\Omega^{A^{\prime} B} K\right), A^{\prime}$ as in $\$ 12$, the above equality can not be asserted.

The following obvious remarks are frequently useful. They will be applied where necessary without further reference. If $\left(\Omega^{o} K^{r}\right)$, and if $Z_{1}, \cdots, Z_{h}$ are the $\Omega$-numbers of degree 1 formed from the columns of the $\Omega$-number $Z$ of degree $h$ in the order in which they occur from left to right, $Z=\left\|Z_{1}, \cdots, Z_{h}\right\|$. Conversely, if $Z$ is of degree $h$, and this equality is given, $Z_{i}$ is the $\Omega$-number of degree 1 formed from the $i$ th column of $Z(i=1, \cdots, h)$. Again, if $X, \cdots, Z$ are any $\Omega^{Q}$-numbers, where $Q$ is either of $P, A P_{2}$, then $\|X, \cdots, Z\|^{\Omega}$ is a symmetric function of $X, \cdots, Z$.

* The first instances of conjunction were given in earlier papers, these Transactions, vol. 22 (1921), p. 1. 
21. Composition as required for the purpose outlined in $\S 7$ is concerned with functions of matric variables. We next define such functions and the relation of equivalence for certain classes of their values.

If whenever the mark $\xi$ and a value $\left(z_{1}^{\prime}, \cdots, z_{r}^{\prime}\right)$ of the matric variable $\left(z_{1}, \cdots, z_{r}\right)$ over $\left(K^{r}\right)$, as defined in $\S 17$, are assigned, there is a unique determination $\xi\left(z_{1}^{\prime}, \cdots, z_{r}^{\prime}\right)$ of the mark $\xi\left(z_{1}, \cdots, z_{r}\right)$, we call $\xi\left(z_{1}^{\prime}, \cdots, z_{r}^{\prime}\right)$ a value of the $\xi$-function $\xi\left(z_{1}, \cdots, z_{r}\right)$ of the argument $\left(z_{1}, \cdots, z_{r}\right)$, or briefly, we say that $\xi\left(z_{1}, \cdots, z_{r}\right)$ is a function of $\left(z_{1}, \cdots, z_{r}\right)$.

Three classes, $F\left(K^{r}\right), F_{r}\left(K^{r}\right), F_{r}$ will be required.

$F\left(K^{r}\right) \equiv$ the class of all functions of the argument $\left(z_{1}, \cdots, z_{r}\right)$.

$F_{r}\left(K^{r}\right) \equiv$ the class of all values of all the functions in $F\left(K^{r}\right)$ generated as $\left(z_{1}, \cdots, z_{r}\right)$ ranges over all its values.

$\xi \equiv$ the class of all values of $\xi\left(z_{1}, \cdots, z_{r}\right)$ in $F_{r}\left(K^{r}\right)$.

$F_{r} \equiv$ the class of all such classes $\xi$.

The notation $F\left(K^{r}\right), F_{r}\left(K^{r}\right), F_{r}$ is henceforth fixed.

If $F_{r} \mid \xi, \zeta$, we say that $\xi, \zeta$ are equivalent, $\xi \sim \zeta$, if and only if $\xi\left(z_{1}, \cdots, z_{r}\right)$ $=\zeta\left(z_{1}, \cdots, z_{r}\right)$ for all values of $\left(z_{1}, \cdots, z_{r}\right)$. It is obvious that equivalence is an instance of abstract equality as defined in $\$ 4$. Hence we may replace $\xi \sim \zeta$ by the usual notation $\xi=\zeta$, bearing in mind, however, that $\xi=\zeta$ is not equality of classes in the sense of mathematical logic. Equivalence implies equality as in mathematical logic, but not conversely.

22. Given now that an operation $\Lambda$ is over $F\left(K^{r}\right)$, we shall define $\Lambda^{o} F_{r}$. Let

$$
\begin{gathered}
\Lambda^{O} F\left(K^{r}\right) \mid \xi\left(z_{1}, \cdots, z_{r}\right), \xi_{i}\left(z_{1}, \cdots, z_{r}\right) \quad(i=1, \cdots, s), \\
\xi\left(z_{1}, \cdots, z_{r}\right)=\left(\xi_{1}\left(z_{1}, \cdots, z_{r}\right), \cdots, \xi_{s}\left(z_{1}, \cdots, z_{r}\right)\right)^{\Lambda} .
\end{gathered}
$$

As $\left(z_{1}, \cdots, z_{r}\right)$ ranges over all its values, the left of the preceding equation generates $\xi$, and the right generates the class of all values of the $\Lambda$-composite of $\left(\xi_{1}\left(z_{1}, \cdots, z_{r}\right), \cdots, \xi_{s}\left(z_{1}, \cdots, z_{r}\right)\right)$; this class will be denoted by $\left(\xi_{1}, \cdots, \xi_{s}\right)^{\Delta}$. Evidently we have

$$
\xi \sim\left(\xi_{1}, \cdots, \xi_{8}\right)^{\Lambda} ;
$$

and therefore by $\S 21$,

$$
\xi=\left(\xi_{1}, \cdots, \xi_{8}\right)^{\Lambda} ; F_{r}\left|\xi_{1}, \cdots, \xi_{s} . \supset . F_{r}\right|\left(\xi_{1}, \cdots, \xi_{8}\right)^{\Lambda} .
$$

From the second of these we may assert $\Lambda^{\circ} F$, where $\Lambda$ as an operation over elements of $F_{r}$ has the meaning just defined for the first. This defines the $\Lambda$-composite $\left(\xi_{1}, \cdots, \xi_{s}\right)$.

23. The nature of the first stage of the theory of composition and inversion for functions of $\Omega$-numbers can now be briefly indicated. The theory is extended in $\$ 50$. 
Given that there exist certain operations under which the class $F_{r}\left(K^{r}\right)$, as in $\$ 21$, is a variety of a prescribed character $(\$ \S 5,12,13)$, and given the like for $F\left(K^{r}\right)$ ( $\left.\$ 21\right)$, we are to construct from some or all of these data operations under which $F_{r}$ is a variety $(\$ \$ 1,21)$.

Suppose that in this way we reach $\Theta^{Q} F_{r}$, where $Q$ is a given character. The problem of inversion then is as follows. Given that $\Theta^{Q} F_{r} \mid \alpha, \beta$, we are to construct $\xi$ such that $\Theta^{Q} F_{r} \mid \xi$ and either $(\alpha, \xi)^{\Theta}=\beta$ or $(\xi, \alpha)^{\boldsymbol{\Theta}}=\beta$.

In constructing $\Theta$ we shall need certain simple properties of total $\Omega$-decompositions. Here we make a remark which need not be repeated, and which obviates a possible duplication of proofs in precisely similar situations. Theorems concerning total $\Omega$-decompositions of the matric variable $\left(z_{1}, \cdots, z_{r}\right)$ over $\left(K^{r}\right)$ can be written down at once by transference from the corresponding theorems for $\Omega$-numbers; see $\$ \$ 18,19$. Again, by transference, any definition relating to $\Omega$-numbers has a unique correspondent for matric variables, and conversely. Hence the theory may be developed with respect either to $\Omega$-numbers or matric variables. The latter is the final form desired, as it is in consonance with properties of functions of $r$ independent variables as usually presented and particularly as customarily written. But when stated in terms of $\Omega$-numbers, the definitions and theorems may be apprehended at a glance, while the transfers are frequently less obvious in appearance. Accordingly we shall state the first forms of all definitions and theorems in terms of $\Omega$-numbers, and later omit such preliminary statements.

24 . Let $\left(\Omega^{A} K^{r}\right)(\$ 17)$. If the $\Omega^{A}$-number $Z$ of degree 1 has $\Omega^{A}$-decompositions of degree $h>1$, the total $\Omega^{A}$-decomposition of degree $h+s-1, s>0$, of $Z$, if it exists, may be obtained as follows. Let $(\$ 20)$

$$
\left\|Z_{1 i}, \cdots, Z_{h-1 i}, Z_{h i}\right\| \quad(i=1, \cdots, p)
$$

be all those $\Omega^{A}$-decompositions of degree $h$ of $Z$ which are such that the $\Omega^{A}$-numbers $Z_{h i}$ of degree 1 have $\Omega^{A}$-decompositions of degree $s$. Let the total $\Omega^{A}$-decomposition of degree $s$ of $Z_{h i}$ be

$$
\left\|Z_{h i 1 p_{i}}, \cdots, Z_{h i s p_{i}}\right\| \quad\left(p_{i}=1, \cdots, q_{i}\right) .
$$

Then, the total $\Omega^{A}$-decomposition of degree $h+s-1$ of $Z$, if it exists, is

$$
\left\|Z_{1 i}, \cdots, Z_{h-1} Z_{h i 1 p_{i}}, \cdots, Z_{h i s p_{i}}\right\| \quad\left(p_{i}=1, \cdots, q_{i} ; i=1, \cdots, p\right) .
$$

For, the $\Omega^{A}$-numbers just written are all distinct, by the definition of a total decomposition in $\$ 15$. The set is exhaustive; otherwise, there would exist an $i$ such that 


$$
\begin{gathered}
X \equiv\left\|Z_{1 i}, \cdots, Z_{h-1_{i}}\right\|, \quad Y \equiv\left\|Z_{h i 1 p_{i}}, \cdots, Z_{h i s p_{i}}\right\|, \\
Y^{\Omega} \equiv W, Z \equiv\|X, W\|, W \neq Z_{h i} ;
\end{gathered}
$$

which is a contradiction, since the decompositions of degree $h$ are total.

If $\left(\Omega^{A} K^{r}\right)$ be weakened to $\left(\Omega^{B} K^{r}\right)$, the weaker hypothesis is insufficient for the above conclusion.

25 . If the $\Omega^{A}$-number $Z$ has $\Omega^{A}$-decompositions of degree $h>1$, it has $\Omega^{A}$-decompositions of degree $t, 0<t<h$. This follows from $\$ \$ 8,20$.

26. As in $\$ \$ 24,25$, we may prove the following. Let

$$
\left\|Z_{1 i}, \cdots, Z_{h i}\right\| \quad(i=1, \cdots, p)
$$

be the total $\Omega^{A}$-decomposition of degree $h$ of the $\Omega^{A}$-number $Z$ of degree 1 , and let the total $\Omega^{A}$-decomposition of degree $n_{j}$ of $Z_{j i}$, if it exists, be

$$
\left\|Z_{j i 1 m_{j}}, \cdots, Z_{j i n_{j} m_{j}}\right\| \quad\left(m_{j}=1, \cdots, t_{j}\right) .
$$

Then, if it exists, the total $\Omega^{A}$-decomposition of degree $n_{1}+\cdots+n_{h}$ of $Z$ is

$$
\begin{aligned}
\left\|Z_{1 i 1 m_{1}}, \cdots, Z_{1 i_{1} m_{1}}, \cdots, Z_{h i 1 m_{h}}, \cdots, Z_{h_{i n_{h} m_{h}}}\right\| \\
\quad\left(i=1, \cdots, p ; m_{j}=1, \cdots, t_{j} ; j=1, \cdots, h\right) .
\end{aligned}
$$

27. In this and the following sections we introduce certain simple tactical considerations indispensable for proofs later. Let $\left(\Omega^{A P_{2}} K^{r}\right)$, and refer to $\$ 8$.

Let $\left\|A_{1}, \cdots, A_{s}\right\|$ be any element of the total $\Omega^{A P_{2}}$-decomposition $S$ of degree $s$ of the $\Omega^{A P_{2}}$-number $Z$ of degree 1 . Then, if precisely $s_{j}$ of $A_{1}, \cdots, A_{s}$ are each equal to $A_{j}^{\prime}(j=1, \cdots, t)$, we have $s_{1}+\cdots+s_{t}=s$, and $\left\|A_{1}, \cdots, A_{s}\right\|$ contributes to $S$ precisely $s ! /\left(s_{1} ! \cdots s_{t} !\right)$ elements, obtained by permuting $A_{1}, \cdots, A_{s}$ in all possible ways.

If in what precedes $\left(\Omega^{A P_{2}} K^{r}\right)$ be replaced by either of the weaker hypotheses $\left(\Omega^{A} K^{r}\right),\left(\Omega^{P_{2}} K^{r}\right)$, the conclusion does not follow.

28. If now $S$ contains further elements, let $\left\|B_{1}, \cdots, B_{s}\right\|$ be one such, and proceed in the same way with this to obtain its total contribution to $S$. Continue this process till $S$ is exhausted $(\$ 16)$, and write all the decompositions under one another, omitting the \|\| from $\left\|A_{1}, \cdots, A_{s}\right\|$, and similarly for the others. In this way we obtain the first of the following arrays or matrices,

$$
\begin{array}{cc}
A_{1}, \cdots, A_{s} & \left(\xi_{1}, A_{1}\right), \cdots,\left(\xi_{s}, A_{s}\right) \\
*, \cdots, * & *, \cdots, * \\
B_{1}, \cdots, B_{s} & \left(\xi_{1}, B_{1}\right), \cdots,\left(\xi_{s}, B_{s}\right) \\
*, \ldots, * & *, \cdots, *
\end{array}
$$

in which the first row of stars indicates all the distinct permutations of 
$A_{1}, \cdots, A_{s}$, except this one, and similarly for the second row of stars and $B_{1}, \cdots, B_{s}$, and so on. The second array is formed from the first by replacing any element $H_{i}$ of the first by $\left(\xi_{i}, H_{i}\right)$, where $\xi_{1}, \cdots, \xi_{s}$ are arbitrary marks. From the construction and the meanings of total decomposition and $\left(\Omega^{A P_{2}} K^{r}\right)$, we have the conclusion next stated.

29. If two columns of the first array in (28) be interchanged, the effect upon the array is at most a permutation of entire rows.

In the second array (28) permute $\left(\xi_{1}, \cdots, \xi_{8}\right)$ or, what is equivalent, perform the same given substitution upon the suffixes $1, \cdots, s$ of $\xi_{1}, \cdots, \xi_{s}$ in every row of the array. Then, since every permutation of $A_{1}, \cdots, A_{s}$, of $B_{1}, \cdots, B_{s}, \cdots$ occurs in the first array (28), and since no permutation of $A_{1}, \cdots, A_{8}$ is a permutation of $B_{1}, \cdots, B_{s}, \cdots$, and similarly for all, we have the second conclusion, stated next.

30. The effect of a permutation of $\xi_{1}, \cdots, \xi_{s}$ in the second array (28) is equivalent to at most a permutation of entire columns of the array followed by a permutation of entire rows.

From this we have the third conclusion, which is the particular one required in composition of functions over classes.

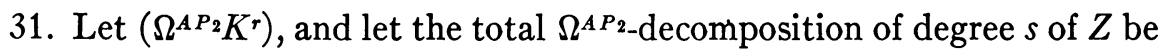
$\left\|Z_{1 i}, \cdots, Z_{s i}\right\|(i=1, \cdots, h)$. Let $\sigma\left(y_{1}, \cdots, y_{s}\right)$ be any symmetric function of the marks $y_{1}, \cdots, y_{s}$. Then a permutation of $\xi_{1}, \cdots, \xi_{s}$ at most permutes the $h$ functions

$$
\sigma\left(\left(\xi_{i}, Z_{1 i}\right), \cdots,\left(\xi_{s}, Z_{s i}\right)\right) \quad(i=1, \cdots, h)
$$

among themselves.

32. In none of what follows is it necessary to postulate that if $\left(\Omega^{A} K^{r}\right) \mid\left(z_{1}, \cdots, z_{r}\right)$, then the matric variable $\left(z_{1}, \cdots, z_{r}\right)$ has $\Omega^{A}$-decompositions of all finite degrees. To take care of the case where decompositions of all finite degrees exist (which, incidentally, is that of all extant theories of numerical functions, except that of Carlitz for finite fields*), we shall state sufficient definitions and conditions for this to be so.

33. An $\Omega$-number $U$ which is such that $\|X, U\|^{\Omega}=X$ for all $\Omega$-numbers $X$, is called an $\Omega$-right modulus of $\left(K^{r}\right)$, and $\left(K^{r}\right)$ is said to be $\Omega$-right modular when $U$ exists. Similarly for left, instead of right, from $\|U, X\|^{\Omega}=X$. If $\|X, U\|^{\Omega}=\|U, X\|^{\Omega}=X$ for all $\Omega$-numbers $X,\left(K^{r}\right)$ is said to be $\Omega$-modular, and we write $\left(\Omega^{M} K^{r}\right)$. If the modulus $U$ is unique, we write $\left(\Omega^{M_{1}} K^{r}\right)$.

If $\left(\Omega^{A P_{2}} K^{r}\right)$ and $\left(K^{r}\right)$ is either right or left modular, then $\left(\Omega^{A P_{2} M} K^{r}\right)$.

If $\left(\Omega^{A} K^{r}\right)$, and if the $\Omega^{A}$-number $X$ has $\Omega^{A}$-decompositions of all finite

* L. Carlitz, American Journal of Mathematics, 1931. 
degrees, $X$ is said to be $\Omega^{A}$-normal. If all $\Omega^{A}$-numbers over $\left(K^{r}\right)$ are $\Omega^{A}$-normal, $\left(K^{r}\right)$ is said to be $\Omega^{A}$-normal, and we write $\left(\Omega^{A N} K^{r}\right)$.

Sufficient conditions that $\left(\Omega^{A N} K^{r}\right)$ are that $\left(\Omega^{A} K^{r}\right)$ and $\left(K^{r}\right)$ have an $\Omega$-right modulus of degree 1 . This condition is not necessary.

Apply the remarks in $\$ 23$ to what precedes.

\section{Composition OF FUnCtions}

34. We now construct an operation $\Theta$ as described in $\$ 23$. Precisely: from the hypotheses

$$
\left(\Omega^{A P_{2} N} K^{r}\right), \quad \Phi O F_{r}\left(K^{r}\right), \Psi^{P} F_{r}\left(K^{r}\right),
$$

in which $\Phi, \Psi$ are arbitrary beyond the properties $O, P$ with respect to $F_{r}\left(K^{r}\right)$ as indicated, we shall produce an interpretation of the matrix $(\Psi, \Phi)$ as an operation $\Theta$ such that $\Theta^{o} F_{r}$. For the notation, we refer to $\$ \S 5,15,17,21,33$, and for equivalent statements of the hypotheses to the general results in $\$ 8$. The whole of $\$ \$ 34-49$ is generalized in $\$ \$ 50-59$.

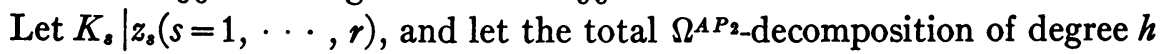
(and necessarily of order 1 ) of $z_{s}$ be

$$
\left(z_{s 1 i_{s}}, \cdots, z_{s h i_{s}}\right) \quad\left(i_{s}=1, \cdots, p_{s}\right) .
$$

Write

$$
Z \equiv\left\|\begin{array}{c}
z_{1} \\
\vdots \\
z_{r}
\end{array}\right\|
$$

Then the total $\Omega^{A P_{2}}$ decomposition of degree $h$ of $Z$ is

$$
\left\|\begin{array}{cc}
z_{11 i_{1}}, z_{12 i_{1}}, \cdots, z_{1 h i_{1}} \\
z_{21 i_{2}}, z_{22 i_{2}}, \cdots, z_{2 h i_{2}} \\
\cdot . \cdot \cdot \cdot \cdot \cdot \cdot \cdot \\
z_{r 1 i_{r}}, z_{r 2 i_{r}}, \cdots, z_{r h i_{r}}
\end{array}\right\| \quad\left(i_{s}=1, \cdots, p_{s} ; s=1, \cdots, r\right)
$$

and the total $\Omega^{A P_{2}-d e c o m p o s i t i o n ~ o f ~ t h e ~ m a t r i c ~ v a r i a b l e ~}\left(z_{1}, \cdots, z_{r}\right)$ is

$$
\left(\left(z_{11 i_{1}}, \cdots, z_{r 1 i_{r}}\right), \cdots,\left(z_{1 h i_{1}}, \cdots, z_{r h i_{r}}\right)\right),
$$

with the same ranges of $i_{1}, \cdots, i_{r}$. Let

$$
F_{r} \mid f_{t} \quad(t=1, \cdots, h) ; p \equiv p_{1} \cdots p_{r} .
$$

Form the $p \Phi$-composites, as permitted by the second hypothesis,

$$
\left(f_{1}\left(z_{11 i_{1}}, \cdots, z_{r 1 i_{r}}\right), \cdots, f_{r}\left(z_{1 h i_{1}}, \cdots, z_{r h i_{r}}\right)\right)^{\Phi} .
$$


The third hypothesis implies that if we now form the $\Psi$-composite of these $p \Phi$-composites, the result is independent of the order in which the $p \Phi$-composites are $\Psi$-composed. Hence this $\Psi$-composite is uniquely known as an element of $F_{r}\left(K^{r}\right)$ when $\Theta \equiv(\Psi, \Phi)$ and the matrix

$$
\left(f_{1}\left(z_{1}, \cdots, z_{r}\right), \cdots, f_{h}\left(z_{1}, \cdots, z_{r}\right)\right)
$$

are assigned, $\Phi, \Psi$ being as in the second and third hypotheses. Hence we have the situation of $\$ 22$ with $\Lambda$ there replaced by $\Theta$, and we may assert $\Theta^{o} F_{r}$.

If $F_{r} \mid f_{1}, \cdots, f_{h}$, we call $\left(f_{1}, \cdots, f_{h}\right)^{\boldsymbol{\Theta}}$, as just constructed, in accordance with the general definitions in $\$ \S 6,22$, the $\Theta$-composite of $\left(f_{1}, \cdots, f_{h}\right)$, with respect to $\left(\Omega^{A P_{2} N} K^{r}\right)$, if necessary to specify the classes $K_{1}, \cdots, K_{r}$ and the properties $A P_{2} N$ of the $\Omega$-numbers constructed from these classes.

We have not used all the implications of the hypothesis $\left(\Omega^{A P_{2} N} K^{r}\right)$ in the construction of $\Theta$. The full restrictions on $\Omega$ are stated from the beginning, as they are required for the derivation of properties of $\Theta$ when $\Phi, \Psi$ are postulated to have further properties. The restriction $N$ is later removed; the postulation of $N$ as above ensures the existence of $\Theta$-composites of all finite degrees $h$.

35. To prove theorems on $\Theta$-composition we refer to the definition of equality (equivalence) in $F_{r}$, stated in $\$ 21$, and proceed as next outlined.

Equality being the only relation which has been defined for elements of $F_{r}$, any theorem concerning $\Theta$-composition must be of the type

$$
\Theta F_{r} \mid f_{1}, \cdots, f_{h}, g_{1}, \cdots, g_{k} . \supset .\left(f_{1}, \cdots, f_{h}\right)^{\Theta}=\left(g_{1}, \cdots, g_{k}\right)^{\Theta}
$$

which is trivially true if the matrices $\left(f_{1}, \cdots, f_{h}\right),\left(g_{1}, \cdots, g_{k}\right)$ are equal. Suppose then that these matrices are unequal. Form the two sets of $\Phi$-composites

$$
\begin{array}{r}
\left(f_{1}\left(z_{11 i_{1}}, \cdots, z_{r 1 i_{r}}\right), \cdots, f_{h}\left(z_{1 h i_{1}}, \cdots, z_{r h i_{r}}\right)\right)^{\Phi} \\
\left(i_{s}=1, \cdots, p_{s} ; s=1, \cdots, r\right) ; \\
\left(g_{1}\left(w_{11 j_{1}}, \cdots, w_{r 1 j_{r}}\right), \cdots, g_{k}\left(w_{1 k j_{1}}, \cdots, w_{r k j_{r}}\right)\right)^{\Phi} \\
\left(j_{s}=1, \cdots, q_{s} ; s=1, \cdots, r\right) ;
\end{array}
$$

the arguments of $f_{1}, \cdots, f_{h}$ being given as in $\$ 34$ from the total $\Omega^{A P_{2} \text {-decom- }}$ position of degree $h$ of $\left(z_{1}, \cdots, z_{r}\right)$, and those of $g_{1}, \cdots, g_{k}$ from the total

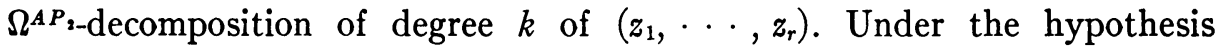
$\Psi^{P} F_{r}\left(K^{r}\right)$, which will be assumed in the theorems considered, the respective $\Psi$-composites of these sets of $\Phi$-composites are significant and the order of $\Psi$-compositions is immaterial. They are uniquely known when $\Theta$ and the matrices

$$
\left(f_{1}\left(z_{1}, \cdots, z_{r}\right), \cdots, f_{h}\left(z_{1}, \cdots, z_{r}\right)\right), \quad\left(g_{1}\left(z_{1}, \cdots, z_{r}\right), \cdots, g_{k}\left(z_{1}, \cdots, z_{r}\right)\right)
$$


are given. If the $\Psi$-composites are equal for all values of $\left(z_{1}, \cdots, z_{r}\right)$, the equality of $\left(f_{1}, \cdots, f_{h}\right)^{\boldsymbol{\Theta}},\left(g_{1}, \cdots, g_{k}\right)^{\boldsymbol{\Theta}}$ is proved. The only question requiring attention is the extent to which postulated properties of $\Phi, \Psi$ carry over to $\Theta$.

Detailed manipulative proofs usually involve complicated multiple suffixes. To avoid such unnecessary complications, we developed the preliminaries in detail, so that the properties of $\Theta$-composition for $\Phi, \Psi$ with assigned properties are immediate consequences of the definitions and $\$ \S 27-31$. If proofs by manipulation are desired, in order to see more clearly the content of the theorems, they may be written out in full by the device of $\$ 10$.

36. If the hypotheses in $\$ 34$ be replaced by

$$
\left(\Omega^{A P_{2} N} K^{r}\right), \Phi^{P} F_{r}\left(K^{r}\right), \Psi^{P} F_{r}\left(K^{r}\right),
$$

then $\Theta^{P} F_{r}$.

This follows from $\S 31$ applied to the construction of $\Theta$ in $\S 34$.

37. If the hypotheses in $\$ 34$ be replaced by

$$
\left(\Omega^{A P_{2} N} K^{r}\right), \Phi^{C P} F_{r}\left(K^{r}\right), \quad \Psi^{C P} F_{r}\left(K^{r}\right),
$$

then $\Theta^{C P} F_{r}$.

38. If the hypotheses in $\$ 34$ be replaced by

$$
\left(\Omega^{A P_{z} N} K^{r}\right), \Phi^{C} F_{r}\left(K^{r}\right), \Psi^{C P} F_{r}\left(K^{r}\right),
$$

then $\Theta^{c} F_{r}$.

39. We wish now to impose sufficient properties upon $\Phi, \Psi$ in $\$ 34$ to ensure $A$ for $\Theta$. It is interesting to observe that $D$ as postulated next is necessary. See $\S 9$ for the notation.

If the hypotheses in $\$ 34$ be replaced by

$$
\left(\Omega^{A P_{2} N} K^{r}\right),(\Psi, \Phi)^{C A P D} F_{r}\left(K^{r}\right),
$$

then $\Theta^{C A P} F_{r}$.

By $\$ \S 8,9$, the second hypothesis may be replaced by

$$
(\Psi, \Phi)^{D} F_{r}\left(K^{r}\right) \text {, and } \Psi^{C P} F_{r}\left(K^{r}\right) \text {, and } \Phi^{C P} F_{r}\left(K^{r}\right),
$$

or by

$$
(\Psi, \Phi)^{D} F_{r}\left(K^{r}\right) \text {, and } \Psi^{C A P_{2}} F_{r}\left(K^{r}\right), \text { and } \Phi^{C A P_{3}} F_{r}\left(K^{r}\right) ;
$$

the conclusion also is equivalent to either of

$$
\Theta^{C P_{2} P_{3} F_{r},} \Theta^{C A P_{2} F_{r} .}
$$

The theorem follows from $\S \S 37,11$, but a proof by manipulation will bring 
out more clearly the nature of the conclusion. By $\$ 37$ it is sufficient to prove $\Theta^{A} F$. Let

$$
\begin{gathered}
\Theta F_{r} \mid \xi_{p}, \xi_{p v_{p}} \\
\xi_{p}=\left(\xi_{p 1}, \cdots, \xi_{p n_{p}}\right)^{\Theta}, \xi=\left(\xi_{1}, \cdots, \xi_{h}\right)^{\Theta},
\end{gathered}
$$

where $h$ is an arbitrary constant integer $>0$. Although it is sufficient to take $h=3$, we shall not impose this restriction. The desired conclusion of the type in $\$ 8$ is here

$$
\begin{aligned}
\boldsymbol{\xi} & =\left(\left(\xi_{11}, \cdots, \xi_{1 n_{1}}\right) \boldsymbol{\Theta}, \cdots,\left(\xi_{h 1}, \cdots, \xi_{h n_{h}}\right)^{\Theta}\right)^{\boldsymbol{\Theta}} \\
& =\left(\xi_{11}, \cdots, \xi_{1 n_{1}}, \cdots, \xi_{h 1}, \cdots, \xi_{h n_{h}}\right)^{\boldsymbol{\Theta}} .
\end{aligned}
$$

Refer to $\$ 26$, and indicate the transfers of $\Omega$-numbers of degree 1 by accents; thus $Z_{1 i}^{\prime}$ is the transfer of $Z_{1 i}$, etc., $Z^{\prime} \equiv\left(z_{1}, \cdots, z_{r}\right)$ is the transfer of the $Z$ in $\$ 26$. We have

$$
\xi=\left(\xi_{1}, \cdots, \xi_{h}\right)^{\boldsymbol{\theta}}
$$

and therefore, by $\$ \$ 26,34$,

$$
\xi\left(Z^{\prime}\right)=\left\{\left(\xi_{1}\left(Z_{11}^{\prime}\right), \cdots, \xi_{h}\left(Z_{h 1}^{\prime}\right)\right)^{\Phi}, \cdots,\left(\xi_{1}\left(Z_{1 s}^{\prime}\right), \cdots, \xi_{h}\left(Z_{h s}^{\prime}\right)\right)^{\Phi}\right\} \Psi .
$$

Similarly, $\xi_{p}\left(Z_{p i}^{\prime}\right)$ is the $\Psi$-composite of the $t_{p} \Phi$-composites

$$
\left(\xi_{p i}\left(Z_{p i 1 m_{p}}^{\prime}\right), \cdots, \xi_{p n_{p}}\left(Z_{p i n_{p} m_{p}}^{\prime}\right)\right)^{\Phi} \quad\left(m_{p}=1, \cdots, t_{p}\right) .
$$

On making the indicated substitutions and applying the general theorem of $\$ 11$ to our present hypotheses, we see that $\xi\left(Z^{\prime}\right)$ is the $\Psi$-composite of the $s t_{1} t_{2} \cdots t_{h} \Phi$-composites

$$
\left\{\xi_{11}\left(Z_{1 i 1 m_{1}}^{\prime}\right), \cdots, \xi_{1 n_{1}}\left(Z_{1 i_{1} m_{1}}^{\prime}\right), \cdots, \xi_{h 1}\left(Z_{h i 1 m_{h}}^{\prime}\right), \cdots, \xi_{h n_{h}}\left(Z_{h i n_{h} m_{h}}^{\prime}\right)\right\}^{\Phi} .
$$

But this is precisely the form of $\xi\left(Z^{\prime}\right)$ that would have been obtained had we proceeded directly from

$$
\left(\xi_{11}, \cdots, \xi_{1 n_{1}}, \cdots, \xi_{h 1}, \cdots, \xi_{h n_{h}}\right)^{\Theta},
$$

calculated for the same argument $Z^{\prime}$. This completes the proof.

40. The chain of theorems in $\$ \$ 34-39$ is completed by the production of a double $(\Psi, \Phi)$-ovum $(\$ 9)$. Later $(\$ \$ 60-63)$ we shall extend the chain, by postulating appropriate moduluses and inverses, to include rings of composition. The following theorem can easily be generalized by weakening the hypotheses, but in the form stated it is closest to extant algebras of numerical functions and includes them. For the notation $F\left(K^{r}\right)$, see $\$ 21$.

If the hypotheses in $\$ 34$ be replaced by

$$
\left(\Omega^{A P_{2} N} K^{r}\right),(\Psi, \Phi)^{C A P D} F_{r}\left(K^{r}\right), \Psi^{A P_{2}} F\left(K^{r}\right),
$$

then $(\Psi, \Theta)^{C A P D} F_{r}$. 
With what has already been proved it is sufficient to show that

$$
\Theta F_{r} \mid \xi, \zeta, \theta . \supset .\left(\xi,(\zeta, \theta)^{\Psi}\right)^{\Theta}=\left((\xi, \zeta)^{\Theta},(\xi, \theta)^{\Theta}\right)^{\Psi},
$$

under the stated hypotheses.

Let $\left(K^{r}\right) \mid z, x_{i}, y_{i}(i=1, \cdots, s)$, and let the total $\Omega^{A P_{2} \text {-decomposition of }}$ degree 2 of the matric variable $z$ of order $r$ be $\left(x_{i}, y_{i}\right)(i=1, \cdots, s)$. The new detail in the proof enters from the third hypothesis. Attending to the definition of $F\left(K^{r}\right)$, we have $\left(\xi(z),(\xi(z), \theta(z))^{\Psi}\right)^{\boldsymbol{\Theta}}=$ the $\Psi$-composite of the $s$ $\Phi$-composites

$$
\left\{\xi\left(x_{i}\right),\left(\zeta\left(y_{i}\right), \theta\left(y_{i}\right)\right) \Psi\right\} \quad(i=1, \cdots, s),
$$

by $\S 34$. But this $\Psi$-composite is equal to the $\Psi$-composite of the two $\Psi$-composites

$$
\begin{aligned}
& \left\{\left(\xi\left(x_{1}\right), \zeta\left(y_{1}\right)\right)^{\Phi}, \cdots,\left(\xi\left(x_{s}\right), \zeta\left(y_{s}\right)\right)^{\Phi}\right\} \Psi \\
& \left\{\left(\xi\left(x_{1}\right), \theta\left(y_{1}\right)\right)^{\Phi}, \cdots,\left(\xi\left(x_{s}\right), \theta\left(y_{8}\right)\right)^{\Phi}\right\} \Psi
\end{aligned}
$$

by the second hypothesis. Hence, since $\left(w^{\Psi}\right)^{\Psi}=w$, the foregoing $\Psi$-composite is the $\Psi$-composite of the $2 s \Phi$-composites

$$
\left(\xi\left(x_{i}\right), \zeta\left(y_{i}\right)\right)^{\Phi},\left(\xi\left(x_{i}\right), \theta\left(y_{i}\right)\right)^{\Phi} \quad(i=1, \cdots, s) .
$$

With a similar reduction of

$$
\left((\xi(z), \zeta(z))^{\Theta},(\xi(z), \theta(z))^{\Theta}\right)^{\Psi},
$$

the proof is completed.

41. An immediate extension of $\$ \$ 34-40$ to the more general situation in which the first hypothesis of $\$ 34$ is replaced by either of

$$
\left(\Omega^{A P_{2}} K^{r}\right),\left(\Omega^{A P_{2} N^{\prime}} K^{r}\right),
$$

is now evident. An integer $h>0$ exists such that there exist $\Omega$-numbers having $\Omega$-decompositions of degree $h$ (we may take $h=1$ if necessary). For a particular $h$ let $S_{h}$ denote the set of all $\Omega^{A P_{1}-n u m b e r s ~ h a v i n g ~} \Omega^{A P_{2}-\text { decompositions of }}$ degree $h$. Replace $N$ in hypothesis 1 by $N_{h}$ and $K^{r}$ by $S_{h}$, where $N_{h}$ indicates

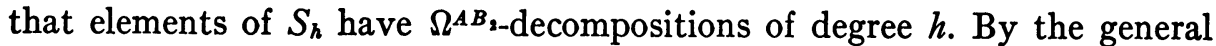

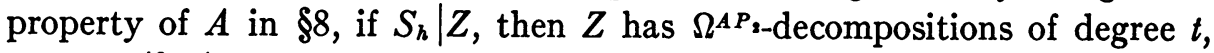
$0<t \leqq h(\S 25)$. Replace $F_{r}\left(K^{r}\right)$ wherever it occurs in the hypotheses by $F_{h}\left(S_{h}\right)$, the class of all values of all functions whose arguments range over $S_{h}$; replace $F\left(K^{r}\right)$ by $F\left(S_{h}\right)$, the class of all functions of $Z^{\prime}$, where $Z^{\prime}$ is the transfer of the general element of $S$. Replace $F_{r}$ by $F_{r}^{\prime}$, defined for $F\left(S_{h}\right)$ precisely as $F_{r}$ was for $F\left(K^{r}\right)$. Replace $P$ wherever it occurs by $P_{h}$, and $A, P_{2}$ by $A_{h}, P_{h}$ which are defined as associativity and commutativity for every $t$ elements $(0<t \leqq h)$. 
Finally replace $\Theta$ by $\Theta_{h}$, which is $\Theta$-composition defined only for classes of functions whose arguments are in $S_{h}$.

What precedes disposes incidentally of the case where at least one of

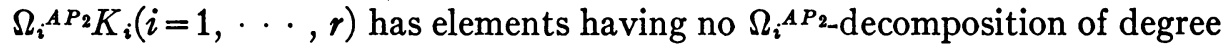
$>h_{i}$, while $K_{i}$ for some $i$ contains elements having $\Omega_{i}{ }^{A P_{2}}$-decompositions of degree $h_{i}$. If $\Omega_{i}{ }^{A P_{2}} K_{i}\left(i=j_{1}, \cdots, j_{p}\right)$ are all such, and if $h$ is the least integer such that $h \geqq h_{u}(u=1, \cdots, p), \Theta_{h}$-composition as above replaces $\Theta$-composition.

42. The object of $\$ \$ 42-48$ is to reduce $\$ \$ 34-41$ to abstract identity with an algebra of numerically valued functions of a single positive integral variable, and to prepare for a complete solution in $\$ \$ 60-63$ of the problem of inversion (\$23). The algebra in question is isomorphic with that of the ring properties of either ordinary Dirichlet series of one variable or ordinary power series of one variable, up to the proviso noted in $\$ 10$. As indicated in $\$ 40$, the proviso will be removed later. Let

$$
\Omega_{j}{ }^{c} K_{j} \mid z_{j}, z_{j 1}, \cdots, z_{j h} ;\left(z_{j 1}, \cdots, z_{j h}\right) \Omega_{i}=z_{j}(j=1, \cdots, r) .
$$

Then, as in $\$ 17,\left(\Omega^{c} K^{r}\right)$, and by the convention in $\S 5$,

$$
\begin{aligned}
& z_{j} \Omega_{j}=z_{j}, \quad z_{j i} \Omega_{j}=z_{j i} \quad(j=1, \cdots, r ; i=1, \cdots, h) ; \\
& \left(\Omega^{C} K^{r}\right) \mid\left(z_{1}, \cdots, z_{r}\right),\left(z_{1 i}, \cdots, z_{r i}\right) \quad(i=1, \cdots, h) \text {, } \\
& \left\|\begin{array}{c}
z_{11}, \cdots, z_{1 h} \\
\cdot . \cdot v^{\Omega} \\
z_{r 1}, \cdots, z_{r h}
\end{array}\right\|^{\Omega}=\left\|\begin{array}{c}
z_{1} \\
\cdot \\
z_{r}
\end{array}\right\| .
\end{aligned}
$$

We now define $\Omega^{\prime} \equiv\left(\Omega_{1}, \cdots, \Omega_{r}\right)$ as an operation such that $\Omega^{\prime}$ is on $\left(K^{r}\right)$, namely $\Omega^{\prime C}\left(K^{r}\right)$, as follows. By definition we put

$$
\left(\left(z_{11}, \cdots, z_{r 1}\right), \cdots,\left(z_{1 h}, \cdots, z_{r h}\right)\right)^{\Omega^{\prime}} \equiv\left(\left(z_{11}, \cdots, z_{1 h}\right) \Omega_{1}, \cdots,\left(z_{r 1}, \cdots, z_{r h}\right) \Omega_{r}\right)
$$

the right of which is known. Hence the left is defined and therefore also $\Omega^{\prime}, \Omega^{\prime O}\left(K^{r}\right)$. We have now

$$
\begin{aligned}
\left(\left(z_{11}, \cdots, z_{r 1}\right), \cdots,\left(z_{1 h}, \cdots, z_{r h}\right)\right)^{\Omega^{\prime}} & =\left(z_{1}, \cdots, z_{r}\right), \\
\left(z_{1}, \cdots, z_{r}\right)^{\prime}=\left(z_{1} \Omega_{1}, \cdots, z_{r} \Omega_{r}\right) & =\left(z_{1}, \cdots, z_{r}\right) .
\end{aligned}
$$

Hence $\left(\Omega^{c} K^{r}\right) . \supset . \Omega^{\prime C}\left(K^{r}\right)$, and obviously $\left(\Omega^{Q} K^{r}\right) . \supset . \Omega^{\prime Q}\left(K^{r}\right)(Q=P, A$, $A P_{2}$ ).

For simplicity in printing we shall denote matric variables and their values over $\left(K^{r}\right)$ by small accented Latin letters, with or without suffixes. Thus, if $K_{i} \mid z_{i}(i=1, \cdots, r)$, we write $z^{\prime}=\left(z_{1}, \cdots, z_{r}\right)$. 
Let the total $\Omega^{c}$-decomposition of degree $h$ of $z^{\prime}(\$ 19)$ be $\left(z_{1 i}^{\prime}, \cdots, z_{h i}^{\prime}\right)$ $(i=1, \cdots, t)$, so that

$$
z^{\prime}=z^{\prime \Omega^{\prime}}=\left(z_{1 i}^{\prime}, \cdots, z_{h i}^{\prime}\right)^{\Omega^{\prime}} \quad(i=1, \cdots, t) .
$$

Then, if $z^{\prime}$ is fixed, the complete solution $\left(x_{1}^{\prime}, \cdots, x_{h}^{\prime}\right)$ of

$$
z^{\prime \Omega^{\prime}}=\left(x_{1}^{\prime}, \cdots, x_{h}^{\prime}\right)^{\prime}
$$

is $\left(x_{1}^{\prime}, \cdots, x_{h}^{\prime}\right)=\left(z_{1 i}^{\prime}, \cdots, z_{h i}^{\prime}\right)(i=1, \cdots, t)$. As definitions of the expressions on the right of the following we now put

$$
\left(x_{1}^{\prime}, \cdots, x_{h}^{\prime}\right) \equiv x_{1}^{\prime} \cdots x_{h}^{\prime}, \quad\left(x_{1}^{\prime}, \cdots, x_{h}^{\prime}\right)^{\Omega^{\prime}} \equiv x_{1}^{\prime} \Omega^{\prime} \cdots x_{h}^{\prime} \Omega^{\prime},
$$

so that $z^{\prime}=x_{1}^{\prime} \cdots x_{h}^{\prime}$ and $z^{\prime \Omega^{\prime}}=\left(x_{1}^{\prime}, \cdots, x_{h}^{\prime}\right)^{\Omega^{\prime}}$ are equivalent statements, and call $x_{1}^{\prime} \cdots x_{h}^{\prime}$ the $\Omega^{\prime}$-product of $x_{1}^{\prime}, \cdots, x_{h}^{\prime}$ in this order. Neither $A$ nor $P$ is yet postulated for $\Omega^{\prime}$.

If $\left(\Omega^{A P_{2}} K^{r}\right)$, then $\Omega^{\prime A P_{2}}\left(K^{r}\right)$, and the $\Omega^{\prime}$-multiplication just defined for $x_{1}^{\prime} \cdots x_{h}^{\prime}$ is both $A$ and $P_{2}$.

43. Scalar multiplication of $\Omega^{\prime}{ }^{A}$-products over $F\left(K^{r}\right), F_{r}\left(K^{r}\right)$ of $\$ 21$ is defined to have all the formal properties of scalar multiplication in a linear associative algebra. For example, if $F_{r}\left(K^{r}\right) \mid p$, the scalar products $p x_{1}^{\prime} \cdots x_{h}^{\prime}$, $x_{1}^{\prime} \cdots x_{h}^{\prime} p$ are equal. Addition of scalar products, indicated by + , is commutative and associative, precisely as in a ring, with the omission of the postulate concerning a modulus and an inverse. Thus the sum of the scalar products $p x_{1}^{\prime} \cdots x_{h}^{\prime}, q y_{1}^{\prime} \cdots y_{k}^{\prime}$ is $p^{\prime} x_{1}^{\prime} \cdots x_{h}^{\prime}+q y_{1}^{\prime} \cdots y_{k}^{\prime} ; p x_{1}^{\prime} \cdots x_{h}^{\prime}$ $+q x_{1}^{\prime} \cdots x_{h}^{\prime}=(p+q) x_{1}^{\prime} \cdots x_{h}^{\prime}$.

Scalar processes have as yet merely a formal or abstract significance. The next sections, on scalar functions, relate scalar processes to $F_{r}$ and $\Theta$.

44. It will be sufficient to develop what follows under the hypotheses of $\$ 40$, as the discussion under weaker hypotheses is precisely similar with the obvious modifications necessary on the $\Gamma$-processes next introduced. These modifications may be inclusively formulated: if $\Psi$ has the character $Q$, then generators (as defined presently) can be combined only so as to preserve the character $Q$ for addition; if $\Phi$ has the character $R$, generators can be combined only so as to preserve the character $R$ for multiplication.

Let $z^{\prime}$ be an arbitrary element (matric variable of order $r$ ) of $\left(K^{r}\right)$. The generator $f_{j}^{\boldsymbol{\Gamma}}$ of $f_{i}$, where $F_{r} \mid f_{j}$, is defined by the following scalar sum of scalar products:

$$
f_{j} \Gamma \equiv \sum f_{j}\left(z_{i}^{\prime}\right) z_{i}^{\prime} \Omega^{\prime} \equiv f_{j}\left(z_{i}^{\prime}\right) z_{1}^{\prime} \Omega^{\prime}+\cdots+f_{j}\left(z_{n}^{\prime}\right) z_{n}{ }^{\prime \Omega^{\prime}}+\cdots,
$$

where the scalar summation $\sum$ refers to all $z_{i}^{\prime}$ such that $\left(K^{r}\right) \mid z_{i}^{\prime}$. By $\$ 16$, the $z_{i}^{\prime}$ are countable, as implied in the above. 
The generators $f^{\Gamma}, g^{\Gamma}$ are said to be equal, $f^{\Gamma}=g^{\Gamma}$, when and only when $f=g$ as in $\$ 21$. Equality of generators is an instance of abstract equality in $\$ 4$. If $f^{\Gamma}=g^{\Gamma}$, the coefficients of $z_{n}^{\prime \Omega^{\prime}}(n=1, \cdots)$ in the two generators are equal, and conversely.

The class of all generators $f^{\Gamma}$ as $f$ ranges over all elements of $F_{r}$ is denoted by $F_{r}^{\prime}$.

45. Under the hypotheses of $\$ 40$ we next define $\Gamma$-composition so that $\Gamma^{c} F_{r}^{\prime}$, and show how $\left(f_{1}, \cdots, f_{h}\right)^{\Gamma}$ may be calculated precisely as is the general coefficient in the formal multiplication of $h$ Dirichlet series in one variable.

Let $f=\left(f_{1}, \cdots, f_{h}\right)^{\boldsymbol{\Theta}}$, as in $\$ 34$. The $\Gamma$-composite $\left(f_{1}, \cdots, f_{h}\right)$ of $\left(f_{1}, \cdots, f_{h}\right)$ is defined to be the generator $f^{\Gamma}$. Hence

$$
\left(f_{1}, \cdots, f_{h}\right) \mathbf{r}=f \mathbf{r}=\sum f\left(z^{\prime}\right) z^{\prime \Omega^{\prime}} ; \quad \Gamma^{C} F_{r}^{\prime} .
$$

Under the stated hypotheses we have also $\Gamma^{A P_{2}} F_{r}^{\prime}$. Further, if $\Sigma^{\prime}$ denotes scalar addition in $F^{\prime}$, defined by

then $\left(\Sigma^{\prime}, \Gamma\right)^{C A P D} F_{r}^{\prime}$.

$$
f \Gamma+g \Gamma \equiv \sum\left(f\left(z^{\prime}\right) g\left(z^{\prime}\right)\right) z^{\prime \prime}{ }^{\prime},
$$

46. For the moment only, let the $z^{\prime}$ denote integers $>0$ and $\Omega^{\prime}$ a real or complex variable. Let $\Pi, \sum$ be as in ordinary analysis. Then

$$
\prod_{j=1}^{h}\left[\sum f_{j}\left(z^{\prime}\right) z^{\prime} \Omega^{\prime}\right]=\sum f\left(z^{\prime}\right) z^{\prime \mathbf{R}^{\prime}},
$$

where the $f_{j}$ are any numerical functions, the summations refer to all $z^{\prime}$, and $f\left(z^{\prime}\right)$ is defined by

$$
f\left(z^{\prime}\right)=\sum f_{1}\left(x_{1 i}^{\prime}\right) \cdots f_{h}\left(x_{h i}^{\prime}\right),
$$

the sum extending to all matrices $\left(x_{1 h}{ }^{\prime}, \cdots, x_{h i}{ }^{\prime}\right)$ of integers $x_{1 h}{ }^{\prime}, \cdots, x_{h i}{ }^{\prime}$ each $>0$ such that $z^{\prime}=x_{1 h}^{\prime} \cdots x_{h i}^{\prime}$. The statement that (46) holds for all integers $z^{\prime}>0$ is written $f=f_{1} \cdots f_{h}$, and the indicated (symbolic) multiplication in $f_{1} \cdots f_{h}$ is associative, commutative and distributive over addition, $f+g$, where $f+g$ is defined by the assertion that $k=f+g$ and $k\left(z^{\prime}\right)=f\left(z^{\prime}\right)+g\left(z^{\prime}\right)$ for all integers $z^{\prime}>0$ are identical statements. For references, see $\$ 56$, footnote.

47. Returning to $\Omega$-numbers, we have now as the equivalent of $\$ 46$ the following abstractly identical situation. Write

$$
\begin{aligned}
& \prod_{j=1}^{h} f_{j}^{\mathbf{r}}=\prod_{j=1}^{h}\left[\sum f_{j}\left(z^{\prime}\right) z^{\prime \Omega^{\prime}}\right]=\sum f\left(z^{\prime}\right) z^{\prime \Omega^{\prime}}, \\
& \prod_{j=1}^{h}\left[\sum f_{i}\left(z^{\prime}\right) z^{\prime} \Omega^{\prime}\right]=\sum\left[\sum f_{1}\left(z_{1 i}^{\prime}\right) \cdots f_{h}\left(z_{h i}^{\prime}\right)\right] z^{\prime} \Omega^{\prime},
\end{aligned}
$$


where the inner $\sum$ in the last has the following interpretation, which automatically interprets the $\Pi$ 's. For each solution $\left(z_{1 i}^{\prime}, \cdots, z_{h i}^{\prime}\right)$ as in $\$ 42$ of $z^{\prime}=x_{1}^{\prime} \cdots x_{h}^{\prime}$ as there, we form the $\Phi$-composite $\left(f_{1}\left(z_{1 i}\right), \cdots, f_{h}\left(z_{h i}\right)\right)^{\Phi}$ and denote the composite by $f_{1}\left(z_{1 i}\right) \cdots f_{h}\left(z_{h i}\right)$. The $\Psi$-composite

$$
\left(f_{1}\left(z_{11}\right) \cdots f_{h}\left(z_{h 1}\right), \cdots, f_{1}\left(z_{1 t}\right) \cdots f_{h}\left(z_{h t}\right)\right) \Psi,
$$

where $t$ is as in $\$ 42$, is now formed and is denoted by $\sum_{i=1}^{t} f_{1}\left(z_{1 i}^{\prime}\right) \cdots \dot{f_{h}}\left(z_{h i}^{\prime}\right)$. With these interpretations of multiplication ( $\Phi$-composition) and addition ( $\Psi$-composition), indicated by the notations customary in a ring or a field, we have

$$
f\left(z^{\prime}\right)=\sum_{i=1}^{t} f_{1}\left(z_{1 i}^{\prime}\right) \cdots f_{h}\left(z_{h i}^{\prime}\right)
$$

Hence, up to the proviso noted in $\$ 10$, there is abstract identity between $\Gamma$-composition of generators and formal multiplication and addition of Dirichlet series of one variable.

48. It follows that theorems concerning $\Theta$-composition are equivalent to identities obtained by equating coefficients of $z^{\prime \mathbf{s}^{\prime}}$ in identities between generators combined according to $\Gamma$-composition $(\$ 45)$. The processes of $\Gamma$-composition can be carried out formally by operating on Dirichlet series as in $\$ 46$ by addition and multiplication, the final results being reinterpreted in terms

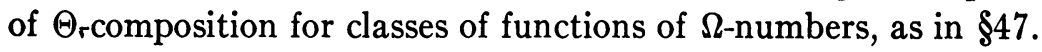

49. In this section we establish a second isomorphism between $\Theta$-composition and the processes of Dirichlet series. It will be shown now that $\Theta$-processes are abstractly identical with those originating in identities obtained by addition and multiplication from Dirichlet series in $r$ independent variables. After the detailed discussion for the case of Dirichlet series in 1 variable, it will be sufficient to present an outline.

For the moment only let $\Omega_{1}, \cdots, \Omega_{r}$ denote independent real or complex variables and consider the following product of $h r$-fold Dirichlet series,

$$
\sum_{j=1}^{h}\left[\sum f_{j}\left(z_{1 j}, \cdots, z_{r j}\right) z_{1 j} \Omega_{1} \cdots z_{r j} \Omega_{r}\right],
$$

where the $\sum$ refers to all values of $z_{1 j}, \cdots, z_{r j}$ ranging independently over all integers $>0$. On reducing this to the form

$$
f \mathrm{r}_{\mathrm{r}} \equiv \sum f\left(z_{1}, \cdots, z_{r}\right) z_{1} \Omega_{1} \cdots z_{r}^{\Omega_{r}},
$$

where the left is to be read $\Gamma_{r}$-generator of $f$, we have

$$
f\left(z_{1}, \cdots, z_{r}\right)=\sum f\left(z_{11 i_{1}}, \cdots, z_{1 h i_{1}}\right) \cdots f\left(z_{r 1 i_{r}}, \cdots, z_{r h i_{r}}\right),
$$


where the $\sum$ refers to all matrices

$$
\left(z_{s i_{s}}, \cdots, z_{s h i_{s}}\right) \quad(s=1, \cdots, r)
$$

of integers $>0$ such that $z_{s}=z_{s 1 i_{\alpha}} \cdots z_{s h i_{s}}$. Compare this with the matrix of $r$ rows and $h$ columns in $\$ 34$.

By an obvious reinterpretation of the notation, as in passing from $\$ 46$ to $\S 47$, we see that the processes of $\Gamma_{r}$-composition of $\Gamma_{r}$ generators, whose formal definition is obvious from what precedes, can be carried out formally by operating on Dirichlet series of $r$ independent variables by addition and multiplication, the final results being translated at once into terms of $\Theta$-composition for classes of functions of $\Omega$-numbers. Further, $\Gamma_{r}$ may replace $\Gamma$ in all conclusions of $\S \S 42-48$.

The modifications necessary if the hypotheses of $\$ 40$ are weakened as in $\$ 41$ are obvious. The abstract identity with power series follows as in Euler algebra (see \$56) by a reinterpretation of the notation.

50. Returning to $\$ 15$, we take advantage of the generality of the definition of $\Omega$-numbers to obtain an indefinite number of generalizations, or iterations, of the theory in $\S \S 34-49$. In $\S 15$ replace $\Omega$ by $i \Omega$, where $i$ is a prefix to distinguish different species of numbers in the sense of $\$ 15$. The class of all ${ }_{i} \Omega$-numbers of given order $s_{i}$ will be denoted by ${ }_{i} K$, and we shall write $\left({ }^{r} K\right) \equiv\left({ }_{1} K, \cdots,{ }_{r} K\right)$. If, for example,

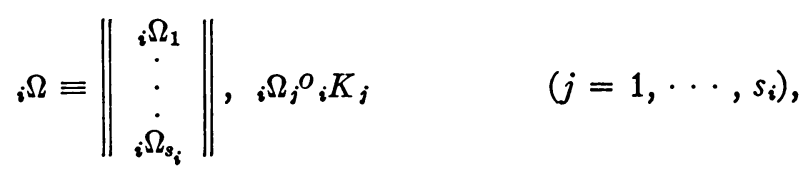

we have merely repeated the definitions in $\$ 15$ with $r, \Omega_{j}, K_{j}$ there replaced by $s_{i},{ }_{i} \Omega_{j},{ }_{i} K_{j}$ here.

We now postulate ${ }_{i} \Omega_{i}{ }_{i} K(i=1, \cdots, r)$. This postulate is satisfied, for example, by conjunction as previously defined. We then define $\bar{\Omega}$ by

$$
\bar{\Omega} \equiv\left\|\begin{array}{c}
1 \Omega \\
\vdots \\
r
\end{array}\right\|
$$

Beginning with $\S 15$, we now replace $\Omega$ by $\bar{\Omega}, K_{j}$ by ${ }_{j} K, \Omega_{j}$ by ${ }_{j} \Omega(j=1, \cdots, r)$, and $\left(K^{r}\right)$ by $\left({ }^{r} K\right)$, up to and including $\S 49$. In $\S 21$ we may change the notation $F_{r}$ to ${ }_{r} F$ wherever $F_{r}$ occurs, and to avoid a possible confusion, transpose all suffixes of elements to prefixes. 
The process just described may be repeated indefinitely. Thus, having just defined $\bar{\Omega}$-numbers, we may replace ${ }_{i} \Omega$ by ${ }_{i} \bar{\Omega},{ }_{i} K$ by ${ }_{i} \bar{K}$, where the last is the class of all ${ }_{i} \bar{\Omega}$-numbers, and proceed in the same way to define $\bar{\Omega}$-numbers where, for example,

$$
\bar{\Omega} \equiv\left\|\begin{array}{c}
1 \bar{\Omega} \\
\vdots \\
: \bar{\Omega}
\end{array}\right\| .
$$

Thus we see that the theory of composition up to $\$ 49$ is indefinitely iterable, and that a given iteration includes all those that precede it, but is not included in any of its predecessors.

51. Each of these iterates in $\$ 50$ is abstractly identical with the algebra of Dirichlet series in one or in $r$ variables, and hence also with the like for power series.

Conversely, it will be seen in $\S 58$, footnote, that the algebras of Dirichlet series and of power series, and the algebras of numerical functions constructed from them, are instances of the algebra of $\Theta$-composition up to $\S 49$.

52. There is another generalization, to functions of $t(t>1)$ independent matric variables over any $t$ classes. We shall merely indicate this, as the detailed development is isomorphic to what precedes and can be obtained by simple changes of notation, or by a reinterpretation of the notation as it is.

In $\S 50$ let $s_{i}=r(i=1, \cdots, t)$, and interpret the conjoint $\Omega^{\dagger}$,

$$
\Omega^{\dagger} \equiv\left\|{ }_{1} \Omega, \cdots,{ }_{t} \Omega\right\|,
$$

as an operator on generalized $\Omega$-numbers $Z^{\dagger}$, where

$$
Z^{\dagger} \equiv\left\|{ }_{1} Z, \cdots, Z\right\|,
$$

and ${ }_{i} Z$ is an ${ }_{i} \Omega$-number of index $\left(r, h_{i}\right)$, by the equation of definition,

$$
Z^{+\Omega^{\dagger}}=\left\|Z_{1} \Omega, \cdots, Z \mathrm{~s}\right\| .
$$

The restatement of the abstract content of $\$ \$ 15-52$ in terms of $\Omega^{\dagger}$-numbers is obvious and will be omitted.

53. Finally, there is the formal generalization of all that precedes to numbers of infinite order. In $\$ 52, t$ need not be finite. It is assumed in the infinite case that the processes are significant. If the classes concerned are modular with respect to the operations, decompositions of infinite degree can also be considered. For instance, see $\S 58$, footnote.

54. The $\Theta$-compositions in what precedes are said to be outer compositions, to distinguish them from inner compositions. In outer composition no law of unique decomposition is postulated, while in inner composition such a 
law, or the abstract equivalent of the fundamental theorem of arithmetic, enters at some stage.

55. The general theory of composition under the stated hypotheses on $\Omega,\left(K^{r}\right), F_{r}\left(K^{r}\right), F\left(K^{r}\right)$ is contained in what precedes. Instances of the theory are constructed in accordance with $\S 1$ by imposing further postulates on one or more of the following sets: $\Psi, \Phi, \Theta ; \Omega,\left(K^{r}\right) ; F\left(K^{r}\right), F_{r}\left(K^{r}\right), F_{r}$. For example, in the third set of possibilities, only the functions in a given subclass of $F\left(K^{r}\right)$ may be $\Theta$-composed, the subclass being such that it is closed under $\Theta$-composition; in the second set, only a subclass of all $\Omega$-numbers need be considered; in the first set, moduluses and inverses for $\Psi$ or $\Phi$ both may be postulated. Finally, examples of any instances so constructed are exhibited by specifying the classes $\left(K^{r}\right), F_{r}\left(K^{r}\right)$. Thus $\left(K^{r}\right)$ may be the class of all one-row matrices of $r$ integers $>0$, and $F_{r}\left(K^{r}\right)$ the class of all functions of $r$ independent variables which are uniform and finite for finite integer values $>0$ of the $r$ variables. As the possibilities are obviously unlimited, we shall outline the development only of that one which includes all types of inner composition, of which there are several instances in the literature.

56. For simplicity we shall state the requisite definitions and postulates in forms which are unnecessarily strong. We shall postulate the hypotheses of $\$ 39$, and we shall use the accent notation of $\$ 42$ for $\Omega^{\prime}, z^{\prime}, \ldots$ with the meanings there explained. By transference $(\$ \$ 18,19)$, the first hypothesis is now $\Omega^{\prime A P_{2} N}\left(K^{r}\right)$. We postulate further that $\left(K^{r}\right)$ is $\Omega^{\prime}$-modular $(\$ 33)$, with the unique modulus $u^{\prime}$. We postulate also that if $f, g$ are any elements of $F_{r}$, then $f\left(u^{\prime}\right)=g\left(u^{\prime}\right)$ and $f\left(u^{\prime}\right)$ (hence also $g\left(u^{\prime}\right), \cdots$ ) is the unique $\Phi$-modulus for $F_{r}\left(K^{r}\right)$.

With respect to $\left(K^{r}\right)$ we now postulate the following: $\left(K^{r}\right)$ contains the subclasses $I\left(K^{r}\right), P\left(K^{r}\right) ; I\left(K^{r}\right) \mid P\left(K^{r}\right)$; and $I\left(K^{r}\right), P\left(K^{r}\right)$ are the maximal classes such that no element of $P\left(K^{r}\right)$ is the $\Omega^{\prime}$-composite of two elements of $P\left(K^{r}\right)$, while each element other than $u^{\prime}$ of $I\left(K^{r}\right)$ not in $P\left(K^{r}\right)$ is, apart from permutations of the components, the $\Omega^{\prime}$-composite of elements of $P\left(K^{r}\right)$ in precisely one way. We have therefore postulated outright the law of unique $\Omega^{\prime}$-decomposition of elements of $I\left(K^{r}\right)$. An element of $I\left(K^{r}\right)$ is called an $\Omega^{\prime}$-integer, an element of $P\left(K^{r}\right)$ an $\Omega^{\prime}$-prime. Two $\Omega^{\prime}$-integers, neither $u^{\prime}$, having $u^{\prime}$ as their unique common $\Omega^{\prime}$-component, are said to be $\Omega^{\prime}$-coprime.

If in the $\Omega^{\prime}$-decomposition $z^{\prime}=x_{1}^{\prime} \cdots x_{h}^{\prime}$ of the $\Omega^{\prime}$-integer $z^{\prime}$ into $\Omega^{\prime}$-primes $x_{1}^{\prime}, \cdots, x_{h}^{\prime}$ (see $\left.\$ 42\right), x_{1}^{\prime}=\cdots=x_{h}^{\prime}$, we write $z^{\prime}=x_{1}^{\prime h}$. Hence, as in rational arithmetic, any $\Omega^{\prime}$-integer $w^{\prime}$ has a unique $\Omega^{\prime}$-decomposition $x_{1}^{\prime h_{1}} \cdots x_{t}^{\prime h_{t}}$, where $x_{1}^{\prime}, \cdots, x_{t}^{\prime}$ are distinct $\Omega^{\prime}$-primes and $h_{1} \cdots h_{t} \neq 0$. By convention, we define $x_{1}^{k^{k_{1}}} \cdots x_{t}^{\prime k_{t}}$, where $k_{1}=\cdots=k_{t}=0$, to be $u^{\prime}$. 
At this stage we may refer to the complete development of the theory of factorable numerical functions of rational integers in previous publications.*

Proceeding as outlined in $\$ 48$, we may reinterpret the whole of the papers referred to in terms of $\left(\Omega^{\prime}, \Phi\right)$-factorable functions, which are defined as follows. If $f\left(\left(x^{\prime}, y^{\prime}\right)^{\Omega^{\prime}}\right)=\left(f\left(x^{\prime}\right), f\left(y^{\prime}\right)\right)^{\Phi}$ whenever $x^{\prime}, y^{\prime}$ are $\Omega^{\prime}$-coprime, we say that $f$ is $\left(\Omega^{\prime}, \Phi\right)$-factorable. In particular, by a mere reinterpretation of a theorem of the previous theory, or independently from the definitions and an application of $\$ 10$, we have the following fundamental theorem for $\left(\Omega^{\prime}, \Phi\right)$ factorable functions.

Let $f, g$ be $\left(\Omega^{\prime}, \Phi\right)$-factorable, and let $(f, g)^{\boldsymbol{\Theta}}=k$. Then $k$ is $\left(\Omega^{\prime}, \Phi\right)$-factorable, and if $z^{\prime}=z_{1}^{\prime a_{1}} \cdots z_{r}^{\prime a_{r}}$ is the $\Omega^{\prime}$-decomposition of the $\Omega^{\prime}$-integer $z^{\prime}$ into powers of distinct $\Omega^{\prime}$-primes $z_{1}^{\prime}, \cdots, z_{r}^{\prime}$, we have

$$
k\left(z^{\prime}\right)=\left(k\left(z_{1}{ }^{\prime a_{1}}\right), \cdots, k\left(z_{r}{ }^{\prime a_{r}}\right)\right)^{\Phi} .
$$

From the last follows at once for $\left(\Omega^{\prime}, \Phi\right)$-factorable functions the whole theory of the Euler product and Euler multiplication, as developed in the publications cited, and the restatement of the previous theory in terms of the present can be made without computations by a simple re-reading of the notation as described in $\$ 48$. In particular, the whole theory of generators, as previously developed, comes over unchanged to the present theory, with the exception (removed later) that reciprocals have (as yet) no existence. Further development in this direction is a matter of unnecessary detail. To the rational unit 1 in the generators of the previous theory (the leading term of the generators there) corresponds here the $\Phi$-modulus as above defined.

57 . The means for developing the theory of $\left(\Omega^{\prime}, \Phi\right)$-factorable functions have just been indicated. In this section and the next we sketch two methods for the construction of further compositions from a given one. By $\$ 48$ we may state the processes in terms of the generators of Euler algebra ( $\$ 7$ of paper cited in \$56), and obtain the corresponding final results here by re-reading the conclusions. A generator in Euler algebra is a power series in the indeterminate $t$ with leading term 1 , in which the coefficients of the several powers of $t$ are arbitrary one-valued functions of the arbitrary constant rational prime $\xi$ (paper cited, p. 148), say $F(t, \xi) \equiv \sum_{0}^{\infty} t^{n} f_{n}(\xi)$. But this generator is equivalent in Euler composition to the one-row matrix $\left(f_{0}(\xi), \cdots\right.$, $\left.f_{n}(\xi), \cdots\right), f_{0}(\xi) \equiv 1$, two such matrices being combined according to Cauchy

* A list of which is given in the Journal of the Indian Mathematical Society, vol. 17 (1928), p. 260. I cite particularly three: University of Washington Publications in Science and Mathematics, vol. 1, No. 1, 1915; Euler algebra, these Transactions, vol. 25 (1923), pp. 135-154; Algebraic Arithmetic, Colloquium Publications of the American Mathematical Society, vol. 7,1927. These contain much more in the present connection than the theory of factorable functions. 
multiplication, namely, the Cauchy product (or composite) of $\left(f_{0}(\xi), \cdots\right.$, $\left.f_{n}(\xi), \cdots\right)$ and $\left(g_{0}(\xi), \cdots, g_{n}(\xi), \cdots\right)$ is $\left(h_{0}(\xi), \cdots, h_{n}(\xi), \cdots\right)$, where

$$
h_{n}(\xi)=\sum_{j=0}^{n} f_{j}(\xi) g_{n-j}(\xi) \quad(n=0,1, \cdots) .
$$

The process just recalled has the characteristic property that it leaves the set of all generators invariant. The function generated by the Cauchy composite as above of two generators is the $E$-composite of the functions generated by the respective generators. In order to secure the properties $A, P_{2}$ for $E$-composition, it is sufficient to compound the generators according to any operation $\Delta$ which leaves the value of the leading term of the compounded generator invariant and has with respect to generators the properties $A, P_{2}$. For example, instead of Cauchy composition as above we may take that in. stance of $\Delta$-composition which is defined by

$$
\left(f_{0}(\xi) g_{0}(\xi), \cdots, f_{n}(\xi) g_{n}(\xi), \cdots\right) .
$$

For any such $\Delta$ the $E$-composite $k$ of $f, g$ is the function generated by the $\Delta$-compound of the generators of $f, g$. To be precise, we therefore refer to the $(\Delta, E)$-composite of $f, g$.

Transferring this to the present theory, we replace $E$ by $\Theta$, and get $(\Delta, \Theta)$-composition. The particular $\Delta$ above becomes here

$$
\left(\left(f_{0}\left(x^{\prime}\right), g_{0}\left(x^{\prime}\right)\right)^{\Phi}, \cdots,\left(f_{n}\left(x^{\prime}\right), g_{n}\left(x^{\prime}\right)\right)^{\Phi}, \cdots\right),
$$

where each of $f_{0}\left(x^{\prime}\right), g_{0}\left(x^{\prime}\right)$ denotes the $\Phi$-modulus (by translating a convention of Euler algebra), and $x^{\prime}$ is an arbitrary constant $\Omega^{\prime}$-prime.

The abstract properties of $\Theta$-composition and $(\Delta, \Theta)$-composition of $\Omega^{\prime}$-factorable functions are identical.

58. Let $K, E$ be any classes, and $\Pi, \Sigma$ operations such that $\Pi^{\circ} K, \Sigma^{o} E$. Let $X, Y, Z, U, \cdots$ denote elements of $K$, and $E(X), E(Y), E(Z), E(U), \cdots$ elements of $E$. The $E(X), \cdots$ are as yet mere marks. If $K, E$ now are such that: whenever $K \mid X$, there is uniquely determined an element, denoted by $E(X)$, of $E$; whenever $E \mid E(X)$, there is uniquely determined an element, denoted by $E^{-1}(E(X))$ or $X$, of $K$;

$$
(X, Y)^{\text {п. } . ~ . ~}(E(X), E(Y))^{\Sigma}
$$

whenever $K \mid X, Y$, and

$$
(E(X), E(Y))^{\Sigma} \text {. د . }(X, Y)^{\Pi}
$$

whenever $E \mid E(X), E(Y)$, we say that $(K, E)$ has $(\Pi, \Sigma)$-correspondence, and call $E(X)$ the (II, $\Sigma$ )-exponent of $X$. 
We shall discuss only the case of $(\Pi, \Sigma)$-correspondence of $(K, E)$ in which ( $\$ 33$ for $M_{1}$ ) $\Sigma^{C A P_{2} M_{1}} E$. Hence, by the above definition, $\Pi^{C A P_{2} M_{1}} K$, and if $U$ is the unique $\Sigma$-modulus of $E$, then $E^{-1}(E(U))$ is the unique $\Pi$ modulus of $K$.

We choose now for $K$ the class $J$ of all $\Omega$-numbers of fixed order $r(\$ 15)$, and take $\Pi \equiv \Omega$. By transference as in $\$ 23$, what follows concerning $(\Omega, \Xi)$ correspondence goes over at once to matric variables over $\left(K^{r}\right)(\$ 17)$ and their exponents, the latter being defined by transference from what is next developed.

The $(\Omega, \Xi)$-exponent of the arbitrary $\Omega$-number $Z$ is now defined for a particular $\Xi$ of great generality. Let

$$
\Xi_{i j}{ }^{C A P_{2} M_{1}} K_{i j} \quad(i=1, \cdots, r ; j=1,2, \cdots),
$$

where the range of $j$ is all finite integers $>0$. A matrix of $r$ rows and an infinity of columns in which the element in row $i$, column $j$, is $a_{i j}$, will be denoted by $\left\|a_{i j}\right\|$, and we need not indicate the ranges $i=1, \cdots, r ; j=1, \cdots$, where these are obvious. The $\Xi_{i j}$ modulus of $K_{i j}$ is denoted by $u_{i j}$, and it is postulated that $u_{i j}$ is $\Xi_{i j}$-indecomposable into two elements of $K_{i j}$ distinct from $u_{i j}$. The matrix $\left\|z_{i j}\right\|$, where $K_{i j} \mid z_{i j}$, is called a $\Xi$-number, where $\Xi \equiv\left\|\Xi_{i j}\right\|$. A $\Xi$-number $\left\|z_{i j}\right\|$ which is such that $z_{i j}=u_{i j}$ for all $j>n$, where $n$ is a finite integer $>0$, is said to be finite. Composition of $\Xi$-numbers is defined by

$$
\left(\left\|x_{i j}\right\|,\left\|y_{i j}\right\|\right) \Xi=\left\|\left(x_{i j}, y_{i j}\right) z_{i j}\right\| .
$$

Hence, by the hypotheses on $\Xi_{i j}$, the $\Xi_{-}$-composite of any finite number of finite $\Xi$-numbers is a finite $\Xi$-number, and if $E$ denotes the class of all finite 艼-numbers,

$$
\Xi^{C A P_{2} M_{1}} E
$$

the unique $\Xi$-modulus of $E$ being $U \equiv\left\|u_{i j}\right\|$.

Precisely as for $\Omega$-numbers we can now define $\Xi$-decomposition. The number of distinct solutions

$$
\left(\left\|x_{i j}\right\|,\left\|y_{i j}\right\|\right) \text { of }\left(\left\|x_{i j}\right\|,\left\|y_{i j}\right\|\right) z=\left\|z_{i j}\right\|,
$$

for $\left\|z_{i j}\right\|$ given, is postulated to be finite (compare $\$ 16$ ). This is equivalent to the postulate in $\$ 16$ for each of $\Xi_{i j}{ }^{o} K_{i j}$.

With $J$ now the class of all $\Omega$-numbers of fixed order $r$ as stated above, and $E$ the class of all finite $\Xi$-numbers, we postulate that each element of $J$ has a finite $(\Omega, \Xi)$-exponent, and we further postulate $(\Omega, \Xi)$-correspondence for $(J, E)$. If $J \mid Z$, the finite $\Xi$-number corresponding to $Z$ is $E(Z)$, and the total $\Omega$-decomposition of degree $h$ of $Z$ is 
where

$$
\left(E^{-1}\left(E\left(Z_{1 i}\right)\right), \cdots, E^{-1}\left(E\left(Z_{h i}\right)\right)\right) \quad(i=1, \cdots, t),
$$

$$
\left(E\left(Z_{1 i}\right), \cdots, E\left(Z_{h i}\right)\right) \quad(i=1, \cdots, t)
$$

is the total $\Xi$-decomposition of degree $h$ of $E(Z)$. Thus the discussion of any question concerning total $\Omega$-decompositions is referred directly to a similar one concerning total $\Xi$-decompositions.

In applying the last to $\Theta$-composition as in $\$ 34$, some care is necessary regarding functions of the transfer $U^{\prime}$ of the $\Xi$-modulus $U$. If $f$ is any element of $F_{r}(\$ 21)$, we postulate now that $f\left(E^{-1}\left(E\left(U^{\prime}\right)\right)\right)$ shall be the $\Phi$-modulus in the $\Theta$-compositions. Or, otherwise: we may first define $f\left(E^{-1}\left(E\left(Z^{\prime}\right)\right)\right)$ to be in $F_{r}\left(K^{r}\right)(\$ 21)$ whenever the transfer $Z^{\prime}$ of $Z$ is in $\left(K^{r}\right)$, and $Z \neq U, F \mid f$, and then define $f\left(E^{-1}\left(E\left(U^{\prime}\right)\right)\right)$ independently as above.*

It is now clear that the whole of $\$ \$ 34-57$ may be re-read with $\Xi$ in place of $\Omega$. There being no restrictions on $\Xi_{i j}, K_{i j}$ beyond those implied in the hypotheses $\Xi_{i j}{ }^{C A P M_{1}} K_{i j}$ and the finiteness of $\Xi_{i j}$-decompositions, the possibilities are again unlimited. The hypotheses can be lightened, as in $\$ 41$. Primality, coprimality and factorability can be defined for $\Xi$-numbers in an obvious manner, following $\$ \$ 56-57$, and then be transferred directly to the corresponding $\Omega$-numbers. Further developments in this direction belong rather to special theories than to the abstract, and we shall not pursue them here, as the postulates for primality are easily realizable in many ways when all the elements of an exponent are either rational numbers or positive rational integers.

* In the instance of numerically valued functions $f\left(n_{1}, \cdots, n_{r}\right)$ of positive rational integers $n_{1}, \cdots, n_{r}$, let, for example,

$$
n_{1}=p_{1}^{z_{11}} p_{2}{ }_{12} \cdots p_{a}^{z_{1 a}}, \cdots, n_{r}=p_{1}^{z_{r 1}} p_{2}{ }^{z_{r 2}} \cdots p_{c}^{{ }^{2} r c},
$$

where the $z$ 's are integers $\geqq 0, p_{1}, p_{2}, \cdots$ the primes $2,3, \cdots$ in ascending order, be the prime factor decompositions of $n_{1}, \cdots, n_{r}$. As one definition of the exponent of the transfer of the matric variable $\left(n_{1}, \cdots, n_{r}\right)$ we may take

$$
\left\|\begin{array}{c}
z_{11}, \cdots, z_{1 a}, 1,1, \cdots \\
\cdot \cdot \cdot \cdot \cdot \\
z_{r 1}, \cdots, z_{r c}, 1,1, \cdots
\end{array}\right\|
$$

and for $\Xi_{i j}$ the operation of multiplication as in rational arithmetic. The $\Xi$-modulus is then the matrix of $r$ rows and an infinity of columns in which every element is 1 . The postulate provides against infinite values of functions whose arguments are the product of all the positive rational primes. In Dirichlet multiplication the 1's in the above exponent (after $z_{1 a}, \cdots, z_{r c}$ ) are replaced by 0 's; the $\Xi_{i j} \equiv$ addition as in rational arithmetic; the $\Xi$-modulus is the matrix of $r$ rows and an infinity of columns, all of whose elements are zero. This instance was first developed in my paper, Bulletin of the American Mathematical Society, vol. 32 (1926), pp. 341-345. The L. C. M.-composition of von Sterneck (Monatshefte für Mathematik, vol. 5 (1894), pp. 255-266) and D. H. Lehmer (American Journal of Mathematics, 1931) has, for $r=1, \Xi_{i j} \equiv$ the operation of replacing a pair of integers by the greater of them. For further instances, see my papers cited in $\$ \S 6,56$, also Annals of Mathematics, vol. 27 (1926), pp. 511-536; American Journal of Mathematics, vol. 49 (1927), pp. 489-510. 
59. When the degree $r$ of the $\Omega$-numbers $(\$ 15)$ in a given context is variable, numerous special properties of $\Theta$-composition arise. As the possibilities are unlimited, we shall consider only one of the simplest: in $\$ 15, r$ is now variable, the classes $K_{1}, \cdots, K_{r}, \cdots$ are taken to be identical, and the operations $\Omega_{1}, \cdots, \Omega_{r}, \cdots$ are the same. To avoid possible confusion we modify the notation; $\left(K^{r}\right)$ of $\$ 17$ now becomes $(K)$.

$(K)$ shall denote the class of all values of all matric variables of all finite orders whose elements are in the class $K$.

For example, $K\left|x_{1}, \cdots, x_{r} . \supset .(K)\right|\left(x_{1}, \cdots, x_{r}\right)$, for all finite integers $r$. Elements of $K$ will be denoted by small Latin unaccented letters, with or without suffixes, elements of $(K)$ by the like accented. Thus $x^{\prime}=\left(x_{1}, \cdots, x_{r}\right)$, etc.

Instead of $F\left(K^{r}\right), F_{r}\left(K^{r}\right)$ of $\$ 21$ we now have $F(K)$, the class of all functions of all matric variables of finite order, and $V(K)$, the class of all values of all functions in $F(K)$. For any fixed $r$, the class $\xi$ in $\$ 21$ is as there defined, and the definition of equality, $\xi=\zeta$, is retained. The class of all $\xi$ is denoted by $F$, which replaces $F_{r}$ of $\$ 21$.

Let $\Lambda, \Xi$ be any operations over $F$, and let $F \mid f, g$ (the $\Xi$ here has no connection with that in $\$ 58)$. The result of operating on $(f, g)$ with $\Lambda$ will be written $(f, g)^{\Delta}$, as before, and the result of operating on $(f, g)^{\Delta}$ (which is in $F$ ) with $\Xi$ will be written $\left((f, g)^{\Lambda}\right)^{\Xi}$, or $(f, g)^{\Lambda \Xi}$. If for all $f, g$ such that $F \mid f, g$, $\Lambda$, $\Xi$ satisfy $(f, g)^{\Lambda \Xi}=(f, g)^{\Xi \Lambda}$, we say that $\Lambda, \Xi$ are permutable over $F$, and write $\Lambda \Xi \Xi \Xi$.

There is an extensive theory of operations permutable over $F$, but as it belongs to the details of the abstract theory, we shall merely exhibit one permutable pair $\Theta, \Upsilon$, where $\Theta$ is the special case of the operation denoted by the same letter in $\$ 34$ which is applicable to the present situation.

We postulate the hypotheses

$$
\triangle^{A P_{2} N} K, \quad(\Psi, \Phi)^{C A P D} V(K),
$$

from which we shall construct $\Theta \equiv(\Psi, \Phi), \Upsilon$ such that

$$
\Theta^{C A P F}, \Upsilon^{O} V(K), \Theta \Upsilon=\Upsilon \Theta .
$$

The $\Delta$ above has no connection with that in $\$ 57$.

As a detail of notation to make what follows clear, if $(f, g)^{\Theta}=h$, we shall indicate the value of $h$ for the argument $z^{\prime}$ by writing $\left(f\left(z^{\prime}\right), g\left(z^{\prime}\right)\right)^{\boldsymbol{\Theta}}=h\left(z^{\prime}\right)$, which accords with $\$ 22$.

It is further postulated, as the equivalent here of $\$ 16$, that if $K \mid x$, the total number of matrices $\left(x_{1}, \cdots, x_{r}\right)$ such that $x=\left(x_{1}, \cdots, x_{r}\right)^{\Delta}$, $K \mid x_{i}(i=1, \cdots, r)$, for each finite integer $r$, is finite. The set of all such 
$\left(x_{1}, \cdots, x_{r}\right)$ is the total $\Delta$-decomposition of degree $r$ of $x$. Order and degree may be used interchangeably in this degenerate case.

The conjoint $\left(x^{\prime}, y^{\prime}\right)$ of $x^{\prime}=\left(x_{1}, \cdots, x_{n}\right), y^{\prime}=\left(y_{1}, \cdots, y_{m}\right)$ is defined as in $\S 20$ to be $\left(x_{1}, \cdots, x_{n}, y_{1}, \cdots, y_{m}\right)$. In particular the conjoint of $x$ and $y^{\prime}$ is $\left(x, y^{\prime}\right)$, where, by the notation already explained, $x$ is an element of $K$ and hence, if preferred, the matrix $(x)$ of one element. If $F \mid f$, the function $f$ for the argument $\left(x^{\prime}, y^{\prime}\right)$ is written $f\left(x^{\prime}, y^{\prime}\right)$ as before.

With $x^{\prime}, y^{\prime}$ as just above, let

$$
\left(f\left(x^{\prime}, y^{\prime}\right), g\left(x^{\prime}, y^{\prime}\right)\right)^{\Theta}=h\left(x^{\prime}, y^{\prime}\right)
$$

for all $\left(x^{\prime}, y^{\prime}\right)$ such that $(K) \mid\left(x^{\prime}, y^{\prime}\right)$. The order (or degree) of the conjoint $\left(x^{\prime}, y^{\prime}\right)$ is here $n+m$. To be explicit, then, the preceding equation may be written

$$
\left(f\left(x^{\prime}, y^{\prime}\right), g\left(x^{\prime}, y^{\prime}\right)\right) \boldsymbol{\Theta}_{(n, m)}=h\left(x^{\prime}, y^{\prime}\right),
$$

where the suffix $(n, m)$ refers to $\left(x^{\prime}, y^{\prime}\right)$ in an obvious manner. The assertion that this equation holds for all $\left(x^{\prime}, y^{\prime}\right)$ such that $(K) \mid\left(x^{\prime}, y^{\prime}\right)$, where the respective orders of $x^{\prime}, y^{\prime}$ are $n, m$, will be written

$$
(f, g) \boldsymbol{\theta}_{(n, m)}=h ;
$$

and finally, the assertion that the last holds for all $(n, m)$ such that $n m>0$ and $n+m$ is finite, will be written $(f, g)^{\boldsymbol{\theta}}=h$. These details are necessary, as we must consider operations which change the orders of the matric variables in the functions.

If $\Theta_{(n, m)}$ has the character $Q$ with respect to the subclass $F_{n+m}$ of $F$ which is generated by all functions of matric variables of order $n+m$ the values of whose elements are in $K$, we write $\Theta_{(n, m)}^{Q} F$. The assertion $\Theta_{(n, m)}^{Q} F$ for all $(n, m)$ as defined is written $\Theta^{Q} F$.

From what precedes and the hypotheses, $\Theta^{C A P} F$.

What follows will be clearer if we describe the structure of $h\left(x^{\prime}, y^{\prime}\right)$ as above in some detail. Write

$$
\left(x^{\prime}, y^{\prime}\right) \equiv z^{\prime} \equiv\left(z_{1}, \cdots, z_{n+m}\right) .
$$

The total $\Delta$-decomposition of degree 2 of $z^{\prime}$ is the class of conjoints of the form $\left(u^{\prime}, v^{\prime}\right)$, a typical pair $u^{\prime}, v^{\prime}$ being

$$
u^{\prime}=\left(u_{1}, \cdots, u_{n+m}\right), v^{\prime}=\left(v_{1}, \cdots, v_{n+m}\right),
$$

in which a particular pair $\left(u_{j}, v_{j}\right)$ is determined as a solution of $\left(u_{j}, v_{j}\right)^{\Delta}$ 
$=z_{j}(j=1, \cdots, n+m)$. For each conjoint $\left(u^{\prime}, v^{\prime}\right)$ thus determined the $\Phi$-composite $\left(f\left(u^{\prime}\right), g\left(u^{\prime}\right)\right)^{\Phi}$ is formed; the $\Psi$-composite of all these $\Phi$-composites, for all $\left(u^{\prime}, v^{\prime}\right)$, is then formed. The result is $h\left(x^{\prime}, y^{\prime}\right)$.

It is important to observe that an alternative construction is possible by $\S 26$. This is to be emphasized, as the permutability of $\Theta, \Upsilon$, established presently, may seem rather abstruse at first sight; at bottom it is precisely the obvious result in $\$ 26$. Applied here, $\$ 26$ enables us to find the total $\Delta$-decomposition of degree 2 of $z^{\prime}$, assumed of order $n+m$, as follows. First write $z^{\prime}$ as the conjoint $\left(x^{\prime}, y^{\prime}\right)$, where $x^{\prime}$ is of order $n$ and $y^{\prime}$ of order $m$. Form the total $\Delta$-decomposition of degree 2 of $x^{\prime}$, and let $\left(x_{i}^{\prime \prime}, x_{i}^{\prime \prime \prime}\right)$ be a typical element of this decomposition. Similarly for $y^{\prime}$ and $\left(y_{j}^{\prime \prime}, y_{j}^{\prime \prime \prime}\right)$. Denote the conjoints $\left(x_{i}^{\prime \prime}, y_{j}^{\prime \prime}\right),\left(x_{i}^{\prime \prime \prime}, y_{j}^{\prime \prime \prime}\right)$ by $z_{i j}^{\prime \prime}, z_{i j}^{\prime \prime \prime}$ respectively. Then the total $\Delta$-decomposition of degree 2 of $z^{\prime}$ is the class of all $\left(z_{i j}^{\prime \prime}, z_{i j}^{\prime \prime \prime}\right)$.

To define $\Upsilon$ we need the intermediary operator $\Upsilon_{x}^{x^{\prime}, n}$, which operates on any $h\left(x^{\prime}, y^{\prime}\right)$ in which $x^{\prime}$ is of fixed order $n, x$ is any given element of $K$, and $y^{\prime}$ any given element of $(K)$. The result of operating on $h\left(x^{\prime}, y^{\prime}\right)$ with $\Upsilon_{x}^{x^{\prime}, n}$ is denoted by $h^{(n)}\left(x, y^{\prime}\right)$, and is obtained as follows: for $x^{\prime}$ is substituted in $h\left(x^{\prime}, y^{\prime}\right)$ in turn each element of the total $\Delta$-decomposition of degree $n$ of $x$, giving, say, the class $h\left(x_{i}{ }^{\prime}, y^{\prime}\right)(i=1, \cdots, t) ; h^{(n)}\left(x, y^{\prime}\right)$ is the $\Psi$-composite of all $h\left(x_{i}^{\prime}, y^{\prime}\right)(i=1, \cdots, t)$. By the hypotheses on $\Psi$, the order of $\Psi$-composition is immaterial. If the order of $y^{\prime}$ is $m$, that of $\left(x^{\prime}, y^{\prime}\right)$ is $n+m$, and the result $f^{(n)}\left(x, y^{\prime}\right)$ of operating with $\Upsilon_{x}^{x^{\prime}, n}$ on $f\left(x^{\prime}, y^{\prime}\right)$ is a function whose argument is of order $1+m$. Hence $\Upsilon_{z}^{z^{\prime, r}}$ operating on $f\left(z^{\prime}\right)$, where $z^{\prime}$ is of order $r$, produces $f^{(r)}(z)$, where the argument is of order 1 , namely $z$ is in $K$. We call $\Upsilon_{x}^{x^{\prime}, n}$ the contraction of $x^{\prime}$ with respect to $x$. This contraction has obvious analogies with that of tensor algebra.

By the above remarks on $\$ 26$ it follows immediately that if $x^{\prime}, y^{\prime}$ are of the respective orders $n, m$, and $f, g, h$ are such that

$$
(f, g)^{\Theta}=h,
$$

then

$$
(f, g)^{\Theta \Upsilon}=(f, g) \Upsilon \Theta,
$$

with the following interpretation:

$$
h^{(n)}\left(x, y^{\prime}\right)=\left(f^{(n)}\left(x, y^{\prime}\right), g^{(n)}\left(x, y^{\prime}\right)\right)^{\Phi(1, m)}
$$

for all $y^{\prime}, n, m$ as defined and all $\Upsilon_{x}^{x^{\prime}, n}$ for all $x$ in $K$. In full, the last equation is $\left[\left(f\left(x^{\prime}, y^{\prime}\right), g\left(x^{\prime}, y^{\prime}\right)\right) \boldsymbol{\Theta}_{(n, m)}\right] \Upsilon_{x}^{x^{\prime}, n}=\left(\left[f\left(x^{\prime}, y^{\prime}\right)\right] \Upsilon_{x}^{x^{\prime}, n},\left[g\left(x^{\prime}, y^{\prime}\right)\right] \Upsilon_{x}^{x^{\prime}, n}\right) \Theta_{(1, m)}$.

Instances of $\Upsilon$ occur in the case of positive integral arguments and the 
special $\Theta$-composition as in the instance by addition in $\$ 58$, footnote, in the papers of Ramanujan and Vaidyanathaswamy.*

\section{INVERSION OF FUNCTIONS}

60. The hypotheses of $\$ 39$ are postulated in $\$ \$ 60-63$, in which we obtain a complete solution of the problem of $\Theta$-inversion as stated in $\$ 23$. It is obvious that a solution without further postulates on $\Omega, \Phi, \Psi,\left(K^{r}\right)$ is impossible. To the postulates carried over from $\$ 39$ we now add the following, in which $M_{1}$ is as in $\$ 33$,

$$
\Omega^{M_{1}}(K), \Psi^{M_{1}} F_{r}\left(K^{r}\right), \Phi^{M_{1}} F_{r}\left(K^{r}\right),
$$

where $\Omega^{\prime}$ is as in $\$ 42$. The first hypothesis of $\$ 39$ is equivalent to $\Omega^{\prime A P_{2} N}(K)$, by the remark in $\$ 23$. Small Latin accented letters shall be as defined in $\$ 42$.

The unique $\Omega^{\prime}$-modulus postulated above for $\left(K^{r}\right)$ will be denoted by $u^{\prime}$, the unique $\Phi$-modulus for $F_{r}\left(K^{r}\right)$ by $\phi$, and the unique $\Psi$-modulus for $F_{r}\left(K^{r}\right)$ by $\psi$.

An $\Omega^{\prime}$-decomposition of $z^{\prime}$ of degree $h$ in which each of the $h \Omega^{\prime}$-components is different from $u^{\prime}$, will be called proper; the set of all proper $\Omega^{\prime}$-decompositions of degree $h$ of $z^{\prime}$ will be called the total proper $\Omega^{\prime}$-decomposition of degree $h$ of $z^{\prime}$.

By $\$ \$ 16,23$, the total proper $\Omega^{\prime}$-decomposition of degree $h$ of $z^{\prime}$, if it exists, contains only a finite number of elements. For simplicity, although it is not necessary, we now impose the following postulate. $\dagger$

Postulate. If $\left(K^{r}\right) \mid z^{\prime}$, then there exists a finite integer $\lambda\left(z^{\prime}\right)$, such that $z^{\prime}$ has at least one proper $\Omega^{\prime}$-decomposition of degree $\lambda\left(z^{\prime}\right)$, and no proper $\Omega^{\prime}$-decomposition of degree $>\lambda\left(z^{\prime}\right)$.

* S. Ramanujan, Transactions of the Cambridge Philosophical Society, vol. 22(1918), p. 260 (=Collected Papers, p. 180); R. Vaidyanathaswamy, Atti del Congresso internazionale dei Matematici (VI), 1928, vol. 2, pp. 105-12; these Transactions, vol. 33 (1931), pp. 579-662. In the last, the instance cited is called convolution, and the author describes the permutability property as a distributivity. The other processes in the paper are as in the references cited in \$56. In the University of Washington publication, 1915, I also described a process which I called "ideal addition," as a complement to "ideal multiplication." Neither, of course, is of the same character as in a field, for in a field the "addition" and "multiplication" must satisfy all the postulates. It seems improper, therefore, to call composition "multiplication," although it satisfies the postulates of multiplication as in a ring, in the fully developed form of the theory. For the same reason, it would seem to be advisable not to speak of the composition in any ring or group as "multiplication." In the book on Algebraic Arithmetic, and in earlier papers, I abandoned the process of "ideal addition" in favor of addition, which has the required distributive properties with respect to "multiplication." Finally, in the present paper, in the interests of complete accuracy, and to avoid any possible misunderstanding, I have dropped the terms "addition," "multiplication" entirely, and have referred to compositions as defined by their respective postulate systems, for example, $\Phi$-composition, $\Psi$-composition, $\Theta$-composition.

$\dagger$ In the instance of rational integers, this amounts to excluding infinity. 
If $z^{\prime} \neq u^{\prime}, z^{\prime}$ has at least one proper $\Omega^{\prime}$-decomposition, namely $z^{\prime},=z^{\prime}$.

Postulate. The $\Omega^{\prime}$-modulus $u^{\prime}$ has no proper $\Omega^{\prime}$-decomposition.*

Two further postulates will be required. The first imposes an additional property on the $\Psi$-modulus $\psi$; the second gives $\Psi$ a unique inverse over $F_{r}\left(K^{r}\right)$.

Postulate. $F_{r}\left(K^{r}\right) \mid f\left(z^{\prime}\right) . \supset .\left(f\left(z^{\prime}\right), \psi\right)^{\Phi}=\psi$.

Postulate. If $F_{r}\left(K^{r}\right) \mid f\left(z^{\prime}\right)$, then there exists a unique element $\tilde{f}\left(z^{\prime}\right)$ of $F(K)$, called the $\Psi$-inverse of $f\left(z^{\prime}\right)$, such that $\left(f\left(z^{\prime}\right), \tilde{f}\left(z^{\prime}\right)\right)^{\Psi}=\psi$, and the following conditions with respect to $\Phi$ are satisfied. If

$$
F_{r}\left(K^{r}\right) \mid g\left(x^{\prime}\right), g\left(y^{\prime}\right) \text { and }\left(f\left(x^{\prime}\right), g\left(y^{\prime}\right)\right)^{\Phi}=h\left(z^{\prime}\right)
$$

(the second of which merely states that the $\Phi$-composite of $\left(f\left(x^{\prime}\right), g\left(y^{\prime}\right)\right)$ is necessarily some element of $\left.F_{r}\left(K^{r}\right)\right)$, then

$$
\left(f\left(x^{\prime}\right), \tilde{g}\left(y^{\prime}\right)\right)^{\Phi}=\tilde{h}\left(z^{\prime}\right),\left(\tilde{f}\left(x^{\prime}\right), \tilde{g}\left(y^{\prime}\right)\right)^{\Phi}=h(z) .
$$

Hence, if $\left(f_{1}\left(x_{1}^{\prime}\right), \cdots, f_{n}\left(x_{n}^{\prime}\right)\right)^{\Phi}=f\left(z^{\prime}\right)$, where $f_{i}\left(x_{i}^{\prime}\right)(i=1, \cdots, n)$ are any elements of $F_{r}\left(K^{r}\right)$, then $\left(\tilde{f}_{1}\left(x_{1}^{\prime}\right), \cdots, \tilde{f}_{n}\left(x_{n}^{\prime}\right)\right)^{\Phi}$ is the element $f\left(z^{\prime}\right)$ or the element $\tilde{f}\left(z^{\prime}\right)$ of $F(K)$ according as $n$ is even or odd. $\dagger$

In the case of functions of rational integers, a distinction between regular and irregular numerical functions is a prerequisite to inversion. The corresponding situation here is provided for by the following definition.

If $F_{r} \mid f$, we say that $f$ is regular if and only if there exists a unique element, which will be denoted by $\bar{f}\left(u^{\prime}\right)$, of $F_{r}\left(K^{r}\right)$, such that $\left(f\left(u^{\prime}\right), \bar{f}\left(u^{\prime}\right)\right)^{\Phi}=\phi$.

In the numerical case of Dirichlet multiplication of functions of one integer variable, $\bar{f}\left(u^{\prime}\right)$ is $1 / f(1)$.

To reach $\Theta$-inversion it is necessary to produce a $\Theta$-modulus $\theta$. If $F_{r}\left(K^{r}\right) \mid \theta\left(z^{\prime}\right)$ whenever $\left(K^{r}\right) \mid z^{\prime}$, we define $\theta$ by

$$
\theta\left(u^{\prime}\right)=\phi, \theta\left(z^{\prime}\right)=\psi\left(z^{\prime} \neq u^{\prime}\right) .
$$

From the construction of $\Theta$ in $\S 34$ and the hypotheses on $\Theta$ assumed at the beginning of this section, it follows that $(f, \theta)^{\Theta}=f$ whenever $F_{r} \mid f$. If there is a second $\Theta$-modulus $\theta^{\prime}$, we have $\left(\theta, \theta^{\prime}\right)^{\Theta}=\theta$, since $\theta^{\prime}$ is a modulus, and $\left(\theta, \theta^{\prime}\right)^{\boldsymbol{\theta}}=\theta^{\prime}$, since $\theta$ is a modulus. But $\left(\theta, \theta^{\prime}\right)^{\boldsymbol{\theta}}$ is a uniquely determined element of $F_{r}$. Hence $\theta^{\prime}=\theta$, and the $\Theta$-modulus is unique.

${ }^{*}$ In the instance of rational numbers, this amounts to restricting the arguments of the functions in the $\Theta$-inverse to be integers.

$\dagger$ No attempt has been made to state an independent set of postulates equivalent to the above. If linear order be postulated for the elements of $(K), \Theta$-inversion can be reached by a shorter route. The way followed here however has the advantage of being immediately applicable to a Boolean algebra or to an abelian group, for the first of which order is not significant and for the second at best artificial. For applications to Boolean algebra, I proceed from my paper on the Arithmetic of logic, these Transactions, vol. 29 (1927), pp. 597-611, in an obvious way. 
61. Let $f$ be any element of $F_{r}$. If an $f^{\prime}$ exists such that $F_{r} \mid f^{\prime}$ and $\left(f, f^{\prime}\right)^{\boldsymbol{\theta}}=\theta$, the equation $(f, g)^{\boldsymbol{\theta}}=h$, where $h$ is any given element of $F_{r}$, has a unique solution $g$ such that $F_{r} \mid g$. For,

$$
\begin{aligned}
(f, g)^{\boldsymbol{\Theta}} & =h . \supset .\left((f, g)^{\boldsymbol{\Theta}}, f^{\prime}\right)^{\boldsymbol{\Theta}}=\left(h, f^{\prime}\right)^{\boldsymbol{\Theta}} ; \\
\left((f, g)^{\boldsymbol{\Theta}}, f^{\prime}\right)^{\boldsymbol{\Theta}} & =\left(g,\left(f, f^{\prime}\right) \boldsymbol{\Theta}\right)^{\boldsymbol{\Theta}}=(g, \theta)^{\boldsymbol{\Theta}}=g ; \\
g & =\left(h, f^{\prime}\right)^{\boldsymbol{\Theta}}, F_{r} \mid g .
\end{aligned}
$$

If $f^{\prime}$ is unique for $f$ given, $g$ is unique, as is seen by a contradiction. If $f^{\prime}$ is not unique, let

$$
\begin{aligned}
\left(f, f^{\prime}\right)^{\boldsymbol{\theta}} & =\theta=\left(f, f^{\prime \prime}\right) \boldsymbol{\Theta}, f^{\prime} \neq f^{\prime \prime} . \\
\left(f^{\prime \prime}, \theta\right)^{\boldsymbol{\theta}} & =\left(f^{\prime \prime},\left(f, f^{\prime}\right) \boldsymbol{\Theta}\right)^{\boldsymbol{\theta}}=\left(\left(f, f^{\prime \prime}\right) \boldsymbol{\Theta}, f^{\prime}\right) \boldsymbol{\Theta}=\left(\theta, f^{\prime}\right) \boldsymbol{\Theta} ; \\
\left(f^{\prime \prime}, \theta\right)^{\boldsymbol{\theta}} & =\left(\theta, f^{\prime}\right)^{\boldsymbol{\theta}} ; \quad f^{\prime \prime}=f^{\prime},
\end{aligned}
$$

a contradiction. Hence $f^{\prime}$ is unique, and therefore if $f^{\prime}$ exists the problem of $\Theta$-inversion is uniquely solvable. We prove next that $f^{\prime}$ exists when and only when $f$ is regular. The proof consists in exhibiting the explicit form of $f^{\prime}$ as a $\Theta$-composite.

62. Let $z^{\prime}$ be any element $\neq u^{\prime}$ of $\left(K^{r}\right)$, and let the total proper $\Omega^{\prime}$-decomposition of degree $k$ of $z^{\prime}$ be $\left(z_{1 i}^{\prime}, \cdots, z_{k i}^{\prime}\right)\left(i=1, \cdots, p_{k}\right)$, where $k \leqq \lambda\left(z^{\prime}\right)$. Let $f$ be any regular element of $F_{r}$. Denote by $\left[f\left(z^{\prime}\right)\right]_{k}$ the following $\Psi$-composite of $p_{k} \Phi$-composites,

$$
\left(\left(\tilde{f}\left(z_{11}^{\prime}\right), \cdots, \tilde{f}\left(z_{k_{1}}^{\prime}\right)\right)^{\Phi}, \cdots,\left(\tilde{f}\left(z_{1 p_{k}}^{\prime}\right), \cdots, \tilde{f}\left(z_{k_{p_{k}}}^{\prime}\right)\right)^{\Phi}\right) \Psi ;
$$

form the $\Phi$-composite of $\left[f\left(z^{\prime}\right)\right]_{k}$ and $k$ components $\bar{f}\left(u^{\prime}\right)$, namely

$$
\left(\left[f\left(z^{\prime}\right)\right]_{k},\left(\bar{f}\left(u^{\prime}\right)\right){ }^{k \Phi}\right) \Phi,
$$

and denote the result by $\left\{f\left(z^{\prime}\right)\right\}_{k}$. Write $\lambda\left(z^{\prime}\right) \equiv n$,

$$
f^{\prime}\left(z^{\prime}\right) \equiv\left(\left\{f\left(z^{\prime}\right)\right\}_{1}, \cdots,\left\{f\left(z^{\prime}\right)\right\}_{n}\right)^{\Psi} .
$$

Define $f^{\prime}\left(u^{\prime}\right)$ by $f^{\prime}\left(u^{\prime}\right) \equiv \bar{f}\left(u^{\prime}\right)$. Then $f^{\prime}$ is the required $\Theta$-inverse of $f$, namely $\left(f, f^{\prime}\right)^{\boldsymbol{\Theta}}=\theta$. Regularity of $f$ is necessary in order that $f^{\prime}$ shall exist; otherwise, $f^{\prime}\left(u^{\prime}\right)$ does not exist.

That $f^{\prime}$ is indeed the required inverse can be easily verified by direct calculation of the value of $\left(f, f^{\prime}\right)^{\boldsymbol{\theta}}$ for the argument $\boldsymbol{z}^{\prime}$. Such a calculation is however unnecessary, as we see that $\left(f, f^{\prime}\right)^{\boldsymbol{\theta}}=\theta$ directly by the method of $\$ 48$ applied to the theorem of inversion for numerical functions of positive rational integers proved in a former paper.* The inversion in that paper and the $\Theta$-inversion here are abstractly identical.

* Tôhoku Mathematical Journal, vol. 17 (1920), pp. 221-225. 
63. We can now remove the exception in $\$ 56$ regarding reciprocals. It is sufficient to combine the hypotheses of $\$ \$ 56,60$ in order to establish complete simple isomorphism between the present theory and that of the papers cited in $\$ 56$. By means of this isomorphism the entire theory previously constructed can be read in terms of the present by a simple reinterpretation of the notation.

64. It was repeatedly remarked in my papers already cited that the elements and operations were abstract, that is, any marks satisfying the postulates. The present paper is, from one point of view, merely an elaboration of that remark. Abstractly, the present theory is identical with my former theory in its simplest form (Euler algebra, as I finally called it, for functions of one variable). The variable there was general, unrestricted beyond the postulates explicitly stated. The abstract point of view has the obvious advantage that inessential details, due to a particular instance, do not obscure the elementary simplicity of the processes involved. It has the disadvantage that an instance may at first sight appear to be a generalization. For example, my extension to functions of $r$ variables* is not a generalization of Euler algebra as developed in the papers cited, but is an instance of that algebra in which the general variable is restricted to be matric of order $r$. The content of the present theory is precisely that of Euler algebra. An instance of this theory can be read as an instance of Euler algebra, by a mere reinterpretation of the notation. If factorability be left out of account, a yet more elementary conclusion emerges: the content of the theory of outer composition ( $\$ 54)$ is identical with that of the formal addition and multiplication of either power series or Dirichlet series in one variable. If factorability in any form be included, the theory (inner composition, \$54) is what it was plus the fundamental theorem of arithmetic.

To generalize, and so reach theories which include Euler algebra or its sub-varieties (algebras $C, D$ of previous papers), it is necessary and sufficient to replace the double $(\Psi, \Phi)$-ovum in $\$ 34$, from which $\Theta$ was constructed, by any variety of which a double $(\Psi, \Phi)$-ovum is an instance. As remarked in $\$ 13$, many such are already known to exist. Those in the literature may be found in the numerous papers of B. A. Bernstein, L. E. Dickson, E. V. Huntington and W. A. Hurwitz on postulate systems, that have appeared in American mathematical periodicals of the past 30 years.

\footnotetext{
* Bulletin of the American Mathematical Society, cited $\S 58$, footnote.

California Institute of Technology, Pasadena, Calir.
} 